\title{
Wirklich nur Mittelmaß? \\ Deutschlands Sozialstaat im Spiegel neuer, international vergleichender Daten
}

von Manfred G. Schmidt

\begin{abstract}
Dieser Beitrag untersucht die Leistungsfähigkeit des deutschen Sozialstaats auf der Grundlage neuer OECD-Vergleichsdaten. Danach erweist sich das deutsche Sozialsystem als ein vielschichtiger Mischtyp aus konservativen, sozialdemokratischen und liberalen Sozialstaatskomponenten. Abgesehen von einer Reihe bedeutender Nebenwirkungen dokumentieren die Daten ein beträchtliches, in der vergleichenden Forschung allerdings weithin unterschätztes Leistungsprofils. Davon zeugen hohe Brutto- und Nettosozialausgaben, weit ausgebaute Mindestsicherungssysteme, die Kombination von Sozialsystemen und einer Regulierung der Arbeitswelt, positive Entwicklungen bei Erwerbs- und Beschäftigungsquoten, Erfolge bei der Bekämpfung von Armut und Einkommensungleichheit sowie die Befähigung zu Sozialstaatsreformen erster, zweiter und dritter Ordnung.
\end{abstract}

This article explores the comparative position of Germany's welfare state on the basis of recent cross-national data. The country's Sozialstaat represents a mixture of conservative, social democratic and liberal components. The data demonstrate both major side-effects of German welfare policies as well as the system's overall high level of performance. The latter is indicated by high levels of gross and net social spending, far-reaching social support structures, a combination of social security with labour market and workplace regulation, increasing labour force participation and employment rates, significant reductions of income inequality and poverty rates, and a variety of welfare state reforms of the first, second and third order.

\section{Einleitung}

Wo steht Deutschlands Sozialstaat im internationalen Vergleich? Erweisen sich die seitens einflussreicher Teile der Komparatistik vorgetragenen Klassifikationen der deutschen Sozialpolitik als zutreffend? ${ }^{1}$ Verkörpert sie wirklich, so die

1 „Sozialstaat“ und weit ausgebaute „Sozialpolitik“ werden im Folgenden als austauschbare und im Wesentlichen dem englischen Begriff des ,,welfare state“ ähnliche Formulierungen gewählt. Im Unterschied zum ,welfare state“ zielt der Sozialstaat allerdings auf sozialen Ausgleich nicht nur durch Systeme der sozialen Sicherung, sondern auch durch Regulierungen der Arbeitswelt - vom Arbeitsschutz über Mitbestimmung bis zum Beschäftigungsschutz. 
eine Variante, einen „Bismarck’schen Sozialversicherungsstaat“‘2, ein im Grunde mittelmäßiges „konservatives Wohlfahrtsstaatsregime“ mit jeweils mittelgradigem Sozialschutz, schwächlicher Umverteilung, unterdurchschnittlichen Erwerbsquoten (insbesondere bei Frauen), relativ hoher sozialer Ungleichheit, Schwächen im Bereich der Armutsbekämpfung, hochgradiger Pfadabhängigkeit sowie begrenzter, deutlich zeitverzögerter Reformfähigkeit? ${ }^{3}$ Oder trifft eine zweite Variante zu, die Deutschlands Wirtschafts- und Sozialpolitik inzwischen auf dem Weg zu einem marktliberalen Modell wähnt? ${ }^{4}$

Der vorliegende Beitrag verneint diese Fragen und gelangt $\mathrm{zu}$ anderen Ergebnissen. Deutschlands Sozialstaat ist deutlich facettenreicher als beide Einordnungen suggerieren und - trotz unbestreitbar schwerer Nebenwirkungen - leistungsstärker als es die Lehre vom „konservativen Wohlfahrtsstaatsregime“ nahelegt. Die Grundlage dieser Diagnose sind neue, insbesondere aus den letzten ein bis zwei Jahrzehnten stammende international vergleichbare Daten zur sozialen Sicherung sowie zur sozialpolitischen Regulierung der Arbeitswelt in den OECDMitgliedstaaten. Diese Daten umfassen neben aktualisierten Zahlen zu den öffentlichen Sozialausgaben der $\mathrm{OECD}^{5}$ neuere Indikatoren aus dem Bereich der öffentlichen und privaten Nettosozialausgaben, ${ }^{6}$ Schätzungen der „Generosität“" von Sozialleistungen, ${ }^{7}$ Befunde aus Spezialuntersuchungen der $\mathrm{OECD}^{8}$ sowie die

2 Jochem, S.: Reformpolitik im deutschen Sozialversicherungsstaat, in: Schmidt, M.G. (Hg.): Wohlfahrtsstaatliche Politik. Institutionen, politischer Prozess und Leistungsprofil, Opladen, 2001, 193-261; Palier, B. (Hg.): A Long Goodbye to Bismarck? The Politics of Welfare Reform in Continental Europe, Amsterdam, 2010.

3 So der Tendenz nach die von G. Esping-Andersen inspirierte Richtung: Esping-Andersen, G.: The Three Worlds of Welfare Capitalism, Cambridge, 1990, $80 \mathrm{ff}$; ders.: Social Foundations of Post Industrial Economies, Oxford, 1999; ders.: The Incomplete Revolution. Adapting to Women's New Roles, Cambridge-Malden, MA, 2009.

4 So aus wirtschaftssoziologischer Sicht Streeck, W.: Re-Forming Capitalism. Institutional Change in the German Political Economy, Oxford, 2009, und in der politikwissenschaftlichen Komparatistik neben Höpner, M./Petring, A./Seikel, D./Werner, B.: Liberalisierungspolitik. Eine Bestandsaufnahme des Rückbaus wirtschafts- und sozialpolitischer Interventionen in entwickelten Industrieländern, in: Kölner Zeitschrift für Soziologie und Sozialpsychologie, 63/1 (2011), 1-32, insbesondere Seeleib-Kaiser, M./van Dyk, S./Roggenkamp, M.: Party Politics and Social Welfare. Comparing Christian and Social Democracy in Austria, Germany and the Netherlands, Cheltenham-Northampton, MA, 2008.

5 OECD: The Social Expenditure Database: An Interpretative Guide SOCX 1980-2007, Paris, 2008.

6 Neuerdings Adema, W./Fron, P./Ladaique, M.: Is the European Welfare State Really More Expensive? Indicators on Social Spending, 1980-2012; and a Manual to the OECD Social Expenditure Database (SOCX) (OECD Social, Employment and Migration Working Papers, No. 124), Paris, 2011.

7 Scruggs, L.: Benefit Generosity Indices, Excel-Datei 2.4.2006 (Abruf Februar 2012); ders.: The generosity of social insurance, 1971-2002, in: Oxford Review of Economic Policy 22/3, 2006, 349-364; ders.: Welfare state generosity across space and time, in: Clasen, J./Siegel, N.A. (Hg.): Investigating Welfare State Change. The 'Dependent Variable Problem' in Comparative Analysis, Cheltenham, UK- 
den Stand der vergleichenden Sozialpolitikforschung porträtierenden Beiträge zum Oxford Handbook of the Welfare State. ${ }^{9}$ Zur politischen Regulierung der Arbeitswelt - eine in der vergleichenden Sozialstaatsforschung häufig vernachlässigte zentrale Dimension der deutschen Sozialpolitik ${ }^{10}$ - liegen inzwischen ebenfalls vergleichbare Daten vor. ${ }^{11}$

\section{Strukturen des Sozialstaats in der Bundesrepublik Deutschland}

Im Rahmen internationaler sozialpolitischer Vergleiche wird häufig nur ein Teil des deutschen Sozialstaats berücksichtigt. Der Großteil der Komparatistik konzentriert sich auf die Sozialversicherungssysteme ${ }^{12}$ und ignoriert etwa die Regulierung der Arbeitswelt sowie die Mindestsicherungssysteme, die in Deutschland derzeit den grundlegenden Lebensunterhalt von 9,3 Prozent der Gesamtbevölkerung finanzieren. ${ }^{13}$ Aus diesem Grund ist es sachdienlich, die Grundzüge des deutschen Sozialstaats nachzuzeichnen, bevor er mit anderen Ländern verglichen wird.

Northampton, MA, 2007, 133-165; ders./Allan, J.: Welfare-state decommodification in 18 OECD countries: a replication and revision, in: Journal of European Social Policy, 16/1, 2008, 55-72; dies.: Social Stratification and Welfare Regimes for the Twenty-first Century: Revisiting The Three Worlds of Welfare Capitalism, in: World Politics, 60/4, 2008, 642-664.

8 Vgl. etwa OECD: Pensions at a Glance - Public Policies across OECD Countries, Paris, 2007; OECD: Pensions at a Glance 2009 - Retirement-Income Systems in OECD Countries, Paris, 2009.

9 Castles, F.G/Leibfried S./Lewis, J./Obinger, H./Pierson, C. (Hg): The Oxford Handbook of the Welfare State, Oxford, 2010.

10 Dies gilt auch für das ansonsten Maßstäbe setzende Oxford Handbook of the Welfare State, a.a.O. Zu den löblichen Ausnahmen gehören Emmenegger, P.: Regulatory Social Policy. The Politics of Job Security Regulations, Bern-Stuttgart-Wien, 2009; Ritter, G.A.: Der Sozialstaat. Entstehung und Entwicklung im internationalen Vergleich, München, 3. Aufl. 2010.

11 Vgl. Emmenegger, P.: a.a.O.; Hörisch, F.: Unternehmensmitbestimmung im nationalen und internationalen Vergleich. Entstehung und ökonomische Auswirkungen, Berlin, 2009; Höpner et al., a.a.O.; OECD: Employment Protection and Labour Market Performance, in: OECD: Employment Outlook, Paris, 1999, 47-132; Siegel, N.A.: Moving beyond expenditure accounts: the changing contours of the regulatory state, 1980-2003, in: Castles, F.G. (Hg.): The Disappearing State? Retrenchment Realities in an Age of Globalisation, Cheltenham-Northampton, MA, 2007, 245-272; Venn, D.: Legislation, collective bargaining and enforcement: Updating the OECD employment protection indicators, www.oecd.org/els/workingpapers, Paris, 2009.

12 Vgl. Alber, J.: Vom Armenhaus zum Wohlfahrtsstaat. Zur Entwicklung der Sozialversicherung in Westeuropa, Frankfurt a.M.-New York, 1982; Esping-Andersen, G.: Three Worlds, a.a.O. und ders.: Social Foundations, a.a.O. sowie die an dens. anknüpfenden Studien von Bambra, C.: Weathering the Storm: Convergence, Divergence and the Robustness of the „Worlds of Welfare“, in: The Social Policy Journal, 3/3 (2004), 3-23 und Scruggs, L./Allan, J.: Welfare-state decommodification, a.a.O.

13 Stand: Jahresende 2008, Bundesamt für Statistik et al. (Hg): Datenreport 2011, Wiesbaden, 2011, 263 f. Zur Struktur und Leistung der Mindestsicherungssysteme: Hauser, R.: Alternativen einer Grundsicherung - soziale und ökonomische Aspekte, in: Wirtschaft - Gesellschaft - Politik 55/3, 2006, 331-348. 
Mit der Sozialpolitik übernimmt Deutschlands Staat „Verantwortung für die Befindlichkeit der Gesellschaft" ${ }^{\star 14}$. Er will dabei, seinen Hauptzielen nach zu urteilen, materielle Verelendung verhindern, Schutz gegen die Wechselfälle des Lebens bieten, soziale Ungleichheit eindämmen, Wohlstand fördern sowie für sozialen Ausgleich auch in der Arbeitswelt sorgen. Hierfür sieht die Sozialpolitik Regelungen vor, die nahezu die gesamte Bevölkerung des Landes erfassen, tief in die Lebens- und Arbeitswelt eingreifen und Sozialleistungen nicht als Almosen verteilen, sondern aufgrund von Rechtsansprüchen der Bürger.

Deutschlands Sozialstaat ist vielschichtig. Er entzieht sich einfachen Typologien. Das gilt auch für die beliebte Unterscheidung dreier „Welten des Wohlfahrtskapitalismus “15 - liberal, konservativ und sozialdemokratisch -, in deren Licht Deutschland den konservativen Typ verkörpert. Dies stellt jedoch ein Zerrbild des deutschen Sozialstaats dar, wie sich anhand seiner fünf Schichten zeigen lässt.

Seine erste und zugleich kostspieligste Schicht sind die Sozialversicherungen. Diese gliedern sich in die gesetzliche Rentenversicherung, auf die derzeit rund 32 Prozent aller öffentlichen Sozialausgaben entfallen, die gesetzliche und private Krankenversicherung (24 Prozent), die Arbeitslosenversicherung (5 Prozent), die Pflegeversicherung (3 Prozent) und die Unfallversicherung (2 Prozent). ${ }^{16}$ Die Leistungen der Sozialversicherungen stehen den beitragszahlenden Arbeitnehmern und ihren nichterwerbstätigen Familienangehörigen zu. Die Sozialversicherungen sind das „Rückgrat" ${ }^{\text {"17 }}$ der Sicherungssysteme hierzulande. Etliche Beobachter stufen Deutschland deshalb als „Sozialversicherungsstaat“" ein.

Doch Sozialpolitik in Deutschland ist mehr als nur „Sozialversicherungsstaat“. Das lehrt allein ein Blick auf die Sozialfinanzen: Rund 65 Prozent der gesamten öffentlichen Sozialausgaben - von Bund, Ländern, Gemeinden und Sozialversicherungen - fallen auf die letztgenannten. Dies bedeutet jedoch, dass 35 Prozent der Ausgaben jährlich in Sozialsysteme jenseits der Sozialversicherungen fließen. Geordnet nach ihrer finanziellen Bedeutung handelt es sich dabei - laut

14 Zacher, H.F.: Grundlagen der Sozialpolitik in der Bundesrepublik Deutschland, in: Bundesministerium für Arbeit und Sozialordnung/Bundesarchiv (Hg.): Grundlagen der Sozialpolitik. Geschichte der Sozialpolitik in Deutschland seit 1945, Bd. 1, Baden-Baden, 2001, 333-684, Zitat 474.

15 Esping-Andersen, G.: Three Worlds, a.a.O.

16 Berechnungsbasis: Bundesministerium für Arbeit und Soziales (BMAS) (Hg.): Übersicht über das Sozialrecht, Nürnberg, 2012. Datenstand: 2010.

17 Ritter, G.A., a.a.O., 192. 
Sozialbudgetsystematik des Bundesarbeitsministeriums von 2010 - um fünf Systeme:

- die „Förder- und Fürsorgesysteme“ (unter ihnen die Grundsicherung für Arbeitssuchende - das sogenannte „Arbeitslosengeld II“ -, sowie Sozialhilfe, Sozialgeld, Kindergeld, Elterngeld, Wohngeld und Leistungen für Asylbewerber),

- die „Arbeitgebersysteme“ (vor allem die Entgeltfortzahlung im Krankheitsfall, die Zusatzversorgung im öffentlichen Dienst und die Betriebliche Altersversorgung),

- die „Systeme des öffentlichen Dienstes“ (Pensionen, Beihilfen und Familienzuschläge),

- die „Sondersysteme“ (neben den berufsständischen Versorgungswerken für Freiberufliche insbesondere die Alterssicherung der Landwirte und die Förderung der privaten Altersvorsorge sowie seit 2010 die Grundleistungen der privaten Krankenversicherung) und

- die „Entschädigungssysteme“ (unter ihnen der Lastenausgleich und die Wiedergutmachung). ${ }^{18}$

Im Unterschied zu den Sozialversicherungen werden diese Systeme - die zweite Schicht des deutschen Sozialstaats - größtenteils aus öffentlichen Haushalten finanziert, mit Ausnahme der Arbeitgebersysteme, der privaten Krankenversicherung und der berufsständischen Versorgungswerke. Ihre Leistungen vergeben all diese Sozialsysteme nach anderen Prinzipien als die Sozialversicherung: Fürsorge (in der Sozialhilfe und beim Sozialgeld), Fördern und Fürsorge (so die Grundsicherung für Arbeitssuchende), Entschädigung (im Sozialschutz für Kriegsopfer), soziale Hilfe (in der Jugendhilfe) und Alimentation (im Falle der standesangemessenen sozialen Sicherung der Berufsbeamten).

Wie in anderen Staaten mit gemischt-konfessioneller Zusammensetzung spielen die Wohlfahrtsverbände in Deutschlands Sozialpolitik ebenfalls eine wichtige Rolle $^{19}$ und stellen eine dritte Schicht dar. Der Schwerpunkt liegt dabei auf der Familien- und der Jugendpolitik, der Pflegeversicherung und der Fürsorge. Die Wohlfahrtsverbände sind Großorganisationen mit rund 1,5 Millionen Teil- oder

19 Schmid, J.: Wohlfahrtsverbände in modernen Wohlfahrtsstaaten. Soziale Dienste in historischvergleichender Perspektive, Opladen, 1996. 
Vollzeitbeschäftigten und etwa einer Million ehrenamtlichen Helfern. ${ }^{20}$ Sie genießen einen Rechtsanspruch auf staatliche Finanzierung - ein ungewöhnliches Privileg.

Die sich damit verbindende Übertragung öffentlicher sozialpolitischer Aufgaben an die Wohlfahrtsverbände auf der Grundlage des Subsidiaritätsprinzips und der Idee des „delegierenden Staates“ stellt in Deutschlands Sozialstaat die vierte Schicht dar. Der subsidiär handelnde Staat überträgt die Verantwortung auf die Eigenhilfe und - bei deren Versagen - in aufsteigender Linie auf Familien- und Nachbarschaftshilfe, gemeinnützige Organisationen und erst dann auf die eigenen Organe. Der „delegierende Staat“ beauftragt zudem Expertengremien mit wichtigen öffentlichen Angelegenheiten, etwa die Leitung der Zentralbank mit der Geldpolitik. Zum ,delegierenden Staat“ zählt zudem die Tarifautonomie: Die verbindliche Regelung von Löhnen und Arbeitsbedingungen obliegt den Tarifparteien, den Interessenvertretungen von Arbeitnehmern und Arbeitgebern, nicht dem Staat.

Schließlich spielt die Regulierung der Arbeitswelt ebenfalls eine wichtige Rolle in Deutschlands Sozialstaat und bildet mithin seine fünfte Schicht. Sie soll für sozialen Ausgleich nicht nur durch Sozialtransfers und soziale Dienstleistungen sorgen, sondern auch durch Mitbestimmungs- und Schutzrechte für die Arbeitnehmer, die Herstellung paritätischer Konfliktbedingungen unter den Tarifparteien sowie die sozialpartnerschaftliche Entschärfung der Interessengegensätze zwischen Arbeit und Kapital. ${ }^{21}$ Zur Regulierung der Arbeitswelt gehört ein anspruchsvoller Arbeits- und Beschäftigungsschutz, eine arbeitnehmerfreundliche Betriebsverfassung und eine ebenfalls weit ausgebaute Arbeitnehmermitbestimmung. ${ }^{22}$ Im Unterschied zur Sozialpolitik der Deutschen Demokratischen Republik und anderer kommunistischer Wohlfahrtsstaaten kennt der Sozialstaat der Bundesrepublik Deutschland kein Äquivalent zur forcierten Vollbeschäftigung. Allerdings ist er zuständig für „Arbeitsförderung“, etwa durch Umschulung und Weiterqualifizierung von Arbeitskräften, sowie für die Verwaltung der Arbeitslosigkeit und ihrer Folgen.

20 Berechnet auf der Basis von Knigge, A.: The Voluntary Welfare Associations in Germany - An Overview, Bremen: Zentrum für Sozialpolitik ZeS-Arbeitspapier Nr. 03/2009.

21 Ritter, G.A., a.a.O.

22 Dittrich, W.: Mitbestimmungspolitik, in: Schmidt, M.G. (Hg.): Die westlichen Länder, München, 1992, 252-260; Hörisch, a.a.O.; Venn, D., a.a.O. 


\section{Deutschlands Sozialstaat im Lichte neuerer international vergleichender Daten und Analysen}

In Deutschland wird mehr Geld für Sozialpolitik ausgegeben als für jedes andere Politikfeld. Auf 30,4 Prozent des Bruttoinlandsprodukts belaufen sich die gesamten öffentlichen Sozialausgaben laut Sozialbudgetstatistik derzeit. ${ }^{23}$ Das entspricht einem Vielfachen der Ausgaben für jeden anderen Politikbereich, etwa fast dem Siebenfachen der öffentlichen Ausgaben für das Bildungswesen. ${ }^{24}$

\section{Brutto- und Nettosozialausgaben}

Von den hohen Sozialausgaben in Deutschland zeugt auch der internationale Vergleich. Deutschland zählt zu jenem kleinen Club von Staaten, in dem der Anteil der öffentlichen Sozialausgaben am Bruttoinlandsprodukt (die sog. Sozialleistungsquote) die 20 Prozent-Marke übersteigt. ${ }^{25}$ Auch im Vergleich mit den wirtschaftlich reicheren Mitgliedstaaten der Organisation für ökonomische Kooperation und Entwicklung (OECD) rangieren Deutschlands Sozialausgaben weit oben. Den OECD-Statistiken zufolge liegt der Anteil der öffentlichen Sozialausgaben am Bruttoinlandsprodukt in Deutschland mit 25,2 Prozent ${ }^{26}$ an sechster Stelle unter den 34 Mitgliedstaaten - knapp hinter Frankreich, Schweden, Österreich, Belgien und Dänemark und weit vor den USA (16,2 Prozent), der Türkei (10,5 Prozent) oder Südkorea (7,6 Prozent). ${ }^{27}$

Zudem wachsen Deutschlands Sozialausgaben weiter - nicht nur absolut, sondern auch relativ: 1960 belief sich die Sozialleistungsquote auf 18,3 Prozent, ${ }^{28}$ 1980 betrug sie 25,7 Prozent und 2010 lag sie um 4,7 Prozentpunkte über dem Stand von 1980.

23 Datenstand 2010. Das entspricht 761 Milliarden Euro. Quelle: BMAS, a.a.O., 1145.

24 4,5 Prozent des Bruttoinlandsproduktes (Datenstand: 2007), OECD: Education at a Glance 2010. OECD Indicators, Paris, 2010, 243.

25 International Labour Office: World Social Security Report 2010/11. Providing coverage in times of crisis and beyond, Genf, 2010, Tabelle 25, $258 \mathrm{ff}$.

26 Diese Zahl ist niedriger als die der deutschen Sozialbudgetstatistik, weil die OECD-Statistik der öffentlichen Bruttoausgaben hauptsächlich zwecks besserer Vergleichbarkeit nicht alle nationalstaatlichen Sozialleistungen erfasst und beispielsweise einen Großteil der steuerfinanzierten und der von Arbeitgebern getätigten Pflichtleistungen nicht berücksichtigt. Ähnliches gilt für die Sozialausgabenstatistik des Statistischen Amtes der Europäischen Union, dessen Schätzung des deutschen Sozialbudgets in etwa denen der OECD entsprechen, vgl. BMAS, a.a.O., $1151 \mathrm{ff}$.

27 Datenstand 2007. S. Tab. 1 im Anhang.

$28 B M A S$, a.a.O., 1144. 
Dass die Sozialausgaben weiter wachsen, mag in einem Zeitalter der Globalisierung und im Lichte hitziger Debatten um ,Sozialabbau“ oder „Demontage des Sozialstaates“ überraschen. Doch das Wachstum der Sozialausgaben stützt einen Befund, den ein gründlicher internationaler Vergleich der Staatsfinanzen schon vor einigen Jahren zu Tage förderte: Die Sozialpolitik zählt in vielen Ländern nicht zu den von Kürzungen besonders hart betroffenen Politikfeldern. ${ }^{29}$ Dies gilt auch für Deutschland - wenngleich es durchaus zu Einschnitten kam. ${ }^{30} \mathrm{Zu}$ den finanziell überdurchschnittlich betroffenen Politikfeldern zählen hingegen hauptsächlich die öffentlichen Investitionen, die Wirtschaftspolitik (v.a. öffentliche Subventionen), die Verteidigung und in etlichen Staaten auch die Bildungspolitik. $^{31}$

Die bislang erwähnten Sozialausgaben sind öffentliche Bruttosozialausgaben. Diese erfassen weder sozialpolitisch gezielte Steuererleichterungen noch die Wirkung der Besteuerung und Sozialabgabenbelastung von Sozialeinkommen oder die gesetzlich vorgeschriebenen privaten Sozialleistungen. Die Bruttosozialausgaben unterschätzen somit einen erheblichen Teil der tatsächlichen Sozialleistungen - in einem von Land zu Land unterschiedlichen Ausmaß. Diese Lücke kann inzwischen jedoch unter Rückgriff auf die sogenannten Nettosozialausgaben geschlossen werden. ${ }^{32}$ Sie erfassen die Steuer- und Sozialabgabeneffekte sowie private Pflichtleistungen, etwa die Lohnfortzahlung im Krankheitsfall, und informieren in der Folge verlässlicher darüber, wie viel von den Sozialleistungen bei den Empfängern nach Abzug von Steuern und Sozialabgaben ankommt.

Eine Betrachtung der Nettosozialausgaben fördert bemerkenswerte Ergebnisse zutage - auch aus deutscher Sicht: Im Lichte der Nettosozialleistungsquote (im oben erwähnten Sinn) ${ }^{33}$ ist Deutschland der nach Frankreich weltweit zweit-

29 Castles, F.G. (Hg.): The Disappearing State? Retrenchment Realities in an Age of Globalisation, Cheltenham-Northampton, MA, 2007. Diesen Befund stützen auch Breunig, C./Busemeyer, M.R.: Fiscal austerity and the trade-off between public investment and social spending, in: Journal of European Public Policy 18/1, 2011, 1-18.

30 Vgl. unter VII.; Siegel, N.A.: Baustelle Sozialstaat. Konsolidierung und Rückbau im internationalen Vergleich, Frankfurt a.M.-New York, 2002.

31 Vgl. die Beiträge zu Castles, F.G. et al., a.a.O.: Cusack, T.R.: Sinking budgets and ballooning prices: recent developments connected to military spending (103-132); Obinger, H./Zohlnhöfer, R.: The real race to the bottom: what happened to economic affairs expenditure after 1980? (184-214) und Schmidt, M.G.: Testing the Retrenchment hypothesis: educational spending, 1960-2002 (159-183).

32 Wegweisend dafür seit Jahr und Tag die Beiträge von Adema, neuerdings Adema, W. et al., a.a.O.

33 Im Sinne der ,net publicly mandated social expenditure“ (Adema, W. et al., a.a.O., Tabelle I.4), der Summe der gesetzlich vorgeschriebenen öffentlichen und privaten Nettosozialleistungen (siehe Tabelle $1 \mathrm{im}$ Anhang). Sie wird - wie auch die anderen Nettosozialleistungsquoten - als Prozentanteil am Brut- 
stärkste Sozialstaat - mit deutlichem Abstand vor Schweden und Dänemark, die bei den Bruttosozialausgaben zu den führenden Staaten zählen. Dort sind aber die Nettosozialleistungsquoten infolge hoher Steuern und hoher Sozialabgaben auf die Sozialeinkommen ihrer Bürger niedriger - 25,9 Prozent in Schweden und 23,9 Prozent in Dänemark.

Adema et al. erfassten überdies die freiwilligen privaten Sozialleistungen. Die Addition dieser Leistungen zu den öffentlichen und den gesetzlich vorgeschriebenen privaten Nettosozialausgaben ergibt die gesamte Nettosozialleistungsquote. Auch hier zählt Deutschland zur Spitzengruppe - erneut vor den skandinavischen Staaten: Mit 28,4 Prozent nimmt Deutschland Rang 3 hinter Frankreich und Belgien ein (vgl. Tab. 1 im Anhang).

Das ist schon für sich bemerkenswert. Der sensationellste Befund betrifft aber Schweden und die USA. Schweden ist bei allen traditionellen Sozialschutzindikatoren eines der führenden Länder - die USA hingegen gehören in der Regel zu den sozialstaatlichen Nachzüglern. Die Nettosozialleistungsquote korrigiert diese Rangordnung drastisch: Was beim Anteil der Bruttosozialausgaben am Bruttoinlandsprodukt ein sehr großer Unterschied war - 32,1 Prozent in Schweden versus 17,4 Prozent in den USA - verschwindet, wenn Steuern, Sozialabgaben sowie gesetzlich vorgeschriebene und freiwillige private Sozialleistungen berücksichtigt werden. Im Licht der gesamten Nettosozialleistungsquote sind die USA mit 27,5 Prozent genauso „sozial“" wie Schweden mit 27,8 Prozent - und auch fast so „,sozial“" wie Deutschland (28,4 Prozent). ${ }^{34}$ Der Grund liegt hauptsächlich in der Zusammensetzung der Sozialausgaben: In den USA sind die Steuern und die Sozialabgaben auf die Sozialeinkommen niedriger als in Deutschland und bedeutend niedriger als in Schweden. Zudem sind die freiwilligen privaten Sozialleistungen, insbesondere die der Unternehmen, in den USA viel höher als in Schweden und Deutschland: In Amerika belaufen sich die freiwilligen privaten Leistungen netto auf 10,1 Prozent des Bruttoinlandsprodukts, in Deutschland auf 1,7 und in Schweden auf 0,6 Prozent. ${ }^{35}$

toinlandsprodukt zu Faktorkosten ausgewiesen. Die Begründung: ,As the construction of net social spending indicators involves adjusting for indirect taxation of consumption out of benefit income, net social expenditure is related to GDP at factor cost, as GDP at factor costs does not include the value of indirect taxation and government subsidies to private enterprises and public corporations" (Adema, $W$. et al., a.a.O., 82).

34 Adema, $W$. et al., a.a.O., Tabelle I.4. Siehe Tabelle 1 im Anhang.

35 Allerdings können gleich große Nettosozialleistungsquoten mit höchst unterschiedlicher Verteilung der Sozialleistungen einhergehen. Die Verteilung der amerikanischen Nettosozialleistungen beispielsweise ist von der schwedischen grundverschieden. Schweden ist ein Staat mit einer vergleichsweise egalitären 
Zurück zu Deutschlands Sozialpolitik und ihrer Position im internationalen Vergleich. Die Auswertung neuerer international vergleichbarer Sozialausgaben führt zu mehreren bemerkenswerten Zwischenergebnissen:

- Weder die Brutto- noch die Nettosozialleistungsquoten in Deutschland verweisen auf ein Abschmelzen der Sozialpolitik.

- Im Vergleich mit allen anderen OECD-Mitgliedstaaten zählt Deutschland bei den Sozialfinanzen, unabhängig von der Messmethode, zur Gruppe der ausgabenstärksten Länder.

- Zudem liegt Deutschlands Nettosozialleistungsquote vor derjenigen der skandinavischen Staaten, die nicht wenigen Sozialstaatskomparatisten als Maß aller sozialpolitischen Dinge gelten.

- Die anhaltend hohen und weiter wachsenden Sozialausgaben stehen quer zu der These, dass der deutsche Sozialstaat in liberale Richtung driftet.

\section{Deutschlands Sozialstaat im Spiegel von Indizes der „Dekommodifizie- rung" und sozialpolitischer Generosität}

Deutschlands Sozialpolitik wird nachgesagt, sie sei bis heute in erster Linie die eines Arbeitnehmersozialstaats geblieben. Dieser kümmere sich hauptsächlich um die Interessen von vollzeitbeschäftigten Beamten, Angestellten und Arbeitern und vernachlässige neue soziale Risiken wie unstete Erwerbskarrieren, hohe Langzeitarbeitslosigkeit, unterdurchschnittlichen Verdienst und Überlastung von erwerbstätigen Alleinerziehenden.

Die These vom Arbeitnehmersozialstaat trifft nicht länger zu. Gewiss: Bis in die 1960er Jahre zielte Deutschlands Sozialstaat vor allem auf die abhängig Beschäftigten und ihren Ruhestand - über lange Strecken mit bevorzugter Behandlung der Beamten, an zweiter Stelle der Angestellten und erst an dritter der Arbeiter. Heutzutage aber schützt Deutschlands Sozialstaat einen viel größeren Personenkreis, tendenziell alle Staatsbürger und faktisch auch den größten Teil der im Lande ansässigen Ausländer, gegen viel mehr Risiken als noch Mitte des 20. Jahrhunderts. ${ }^{36}$ Zudem werden heutzutage Sozialtransfers, wie Renten oder Ar-

Sozialpolitik für tendenziell alle Staatsbürger. Die amerikanische Sozialpolitik hingegen ist durch stärkere Konzentration und auffällige Schichtung der Sozialleistungen gekennzeichnet. So profitieren von den privaten Sozialausgaben in großem Maße die regulär Beschäftigten in mittleren und großen Betrieben, vgl. Seeleib-Kaiser, M.: Globalisierung und Sozialpolitik. Ein Vergleich ihrer Diskurse und Wohlfahrtssysteme in Deutschland, Japan und in den USA, Frankfurt a.M.-New York, 2001. Und trotz hoher öffentlicher und privater Ausgaben für Gesundheit haben mehrere 10 Millionen Amerikaner keinen Krankenversicherungsschutz.

36 Vgl. Bundesministerium für Arbeit und Sozialordnung/Bundesarchiv, a.a.O.; BMAS, a.a.O. 
beitslosengeld, in viel größerem Maße als früher durch soziale Dienstleistungen ergänzt. Davon zeugen der Aufbau der Pflegeversicherung, die Familienpolitik sowie die sozialen Dienstleistungen im Rahmen der Arbeitsmarktpolitik und im Gesundheitswesen. Der Sozialschutz reicht überdies weit über den Kreis der abhängig Beschäftigten hinaus, wie allein die Vielzahl der unter II. erwähnten Sozialsysteme zeigt. In der Summe erfassen diese Systeme inzwischen nahezu die gesamte Wohnbevölkerung in Deutschland. Überdies decken sie den Großteil jener Risiken, die die Vorsorgekraft des Einzelnen meist übersteigen, mehr oder minder zuverlässig ab.

Ferner sind zwei Aspekte zu bedenken, die im Rahmen internationaler Vergleiche der Sozialpolitik meist vernachlässigt werden: Deutschlands Mindestsicherungssysteme schützen jeden Bedürftigen vor materieller Verelendung. Das ist ein grundlegender Unterschied zur amerikanischen Sozialpolitik, die nicht vor absoluter Armut schützt, sondern im eigenen Land den Absturz gleichsam in die Dritte Welt durchaus zulässt. Mindestsicherungssysteme sind im deutschen Fall, so sei der Vollständigkeit halber ergänzt, ein komplexes Arrangement, das sich aus Sozialhilfe, Sozialgeld, Arbeitslosengeld II, Kriegsopferfürsorge, Grundsicherung im Alter und bei Erwerbsminderung sowie Leistungen nach dem Asylbewerberleistungsgesetz zusammensetzt. Vernachlässigt wird im internationalen Vergleich meist auch ein weiterer Pfeiler des Sozialstaats: die politische Regulierung der Arbeitswelt (vgl. unter IV.).

Das Zusammenspiel von Sozialversicherungen und Mindestsicherungssystemen schützt die Bevölkerung in Deutschland vor materieller Verelendung und bewahrt sie davor, wie eine Ware behandelt zu werden und den Lebensunterhalt ausschließlich aus Beschäftigungsverhältnissen zu finanzieren. Die Sozialpolitik bringt insoweit „Dekommodifizierung“ zustande, so der von Esping-Andersen geprägte Fachbegriff für den Schutz der Sozialpolitik vor kompletter Marktabhängigkeit. ${ }^{37}$ Das Ausmaß der Dekommodifizierung unterscheidet sich, EspingAndersen zufolge, je nach Typ des Wohlfahrtsstaats: Es ist gering im liberalen, mittelgradig im konservativen und hochgradig im sozialdemokratischen Wohlfahrtsstaat. ${ }^{38}$

37 Esping-Andersen, G.: Three Worlds, a.a.O. Zugleich sorgt aber die deutsche Sozialpolitik auch für Arbeitsförderung und für Arbeitsmarkteingliederung von Personen im erwerbsfähigen Alter. Sozialpolitik hat demnach auch mit „Kommodifizierung“ zu tun, vgl. die Kapitel 4 und 5.

38 Ebd. und Esping-Andersen, G.: Social Foundations, a.a.O. zufolge sind die USA das Paradebeispiel des liberalen Wohlfahrtsstaats; Deutschland stehe für den konservativen und Schweden für den sozialdemokratischen Typ. 
Bei der Erstmessung in Esping-Andersens ,Three Worlds of Welfare Capitalism " wurde Deutschland eine mittelgradige Dekommodifizierung bescheinigt gemessen an verschiedenen Indikatoren der Renten-, Kranken- und Arbeitslosenversicherung Anfang der 1980er Jahre. Dieser Messung zufolge nahm die deutsche Sozialpolitik im internationalen Vergleich eine Position in der Mitte ein - weit vor den USA und mit erheblichem Abstand hinter den skandinavischen Ländern (s. Tab. 1 im Anhang).

Esping-Andersen schrieb seinen Index der Dekommodifizierung nicht fort - auch nicht in seinen neueren Studien. ${ }^{39}$ Inzwischen gibt es allerdings eine Alternative: den Indikator der „Generosität“ von Sozialleistungen, den Scruggs auf Jahresbasis für 18 OECD-Mitgliedstaaten im Zeitraum von 1971 bis 2002 konstruierte. $^{40}$ Die „Generosität“ der Sozialleistungen ermittelt Scruggs im Anschluss an Esping-Andersens Studie im Wesentlichen anhand dreier Sozialprogramme - Rentenversicherung, Arbeitslosenversicherung und Krankengeld (sick payment) und dreier Dimensionen: der Nettolohnersatzrate ${ }^{41}$, des Deckungsgrades der Programme (coverage) - gemessen an der Größe des erfassten Personenkreises und der Restriktionen im Zugang zu den Sozialleistungen, etwa durch Wartezeiten. Die Messergebnisse addiert Scruggs zum „Generositäts-Index“ (overall generosity score) - einem Anzeiger der gesamten sozialpolitischen „Großzügigkeit". 42

Diesen Index wertet Scruggs als die bessere Alternative zu dem der Dekommodifizierung ${ }^{43}$ - mit einigem Recht, wenngleich beide einige ähnliche Schwächen aufweisen. $^{44}$ Für Scruggs' Generositäts-Index sprechen die im Jahresabstand

39 Esping-Andersen, G.: Three Worlds, a.a.O; ders.: Incomplete Revolution, a.a.O.

40 Scruggs, L.: The Generosity of Social Insurance, a.a.O.; ders.: Welfare State Generosity, a.a.O.; ders./Allan, J.: Welfare-state decommodification, a.a.O.

41 Sie wird auf Jahresbasis für zwei Haushaltstypen ermittelt: eine allein wohnende ledige Person und ein Paar mit einem Verdiener und zwei Kindern (oder ohne Kinder, so im Falle der Renten).

42 Scruggs, L.: Preliminary Codebook Welfare State Entitlements: A Comparative Institutional Analysis of Eighteen Welfare States, Version 1.1, June 1, 2005.

43 Ders.: Benefit Generosity Indices, Excel-Datei 2.4.2006 (Abruf Februar 2012); ders.: Welfare State Generosity, a.a.O.; ders./Allan, J. 2008, Welfare-state decommodification, a.a.O.

44 Dass obendrein alternative Messungen der Sozialleistungen, insbesondere die Social Rights-Messungen von Palme, J./Korpi, W.: New Politics and Class Politics in the Context of Austerity and Globalisation: Welfare State Regress in 18 Countries, 1975-95, in: American Political Science Review, 97/2, 2003, 425-446 mitunter erheblich von Scruggs' Daten abweichen, gehört zu den noch weiter zu klärenden Aufgaben der vergleichenden Sozialpolitikforschung. Vgl. Wenzelburger, G./Zohlnhöfer, R./Wolf, F.: Replacement Rate Roulette. Implications of Dataset Choice in Comparative Welfare State Research, Heidelberg, unveröffentlichtes Manuskript, 2012. Ein Teil der Unterschiede kommt laut Wenzelburger, G. et al., a.a.O. dadurch zustande, dass Scruggs im Unterschied zu Korpi, W./Palme, J. die Bedürftig- 
erfolgenden Messungen sowie die genauere Berücksichtigung von Steuern und Sozialabgaben auf die Sozialleistungen. Zu den Schwächen des GenerositätsIndexes zählt allerdings, dass auch er weder die Mindestsicherungssysteme erfasst, noch die Pflegeversicherung oder die flächendeckenden Gesundheitsdienstleistungen unabhängig von der Höhe der Versicherungsbeiträge der Versicherten - ganz zu schweigen von der sozialpolitischen Regulierung der Arbeitswelt. Zudem unterschätzt Scruggs, wie schon Esping-Andersen, die Generosität der deutschen Rentenversicherung. Das hat mehrere Gründe: Berücksichtigt werden nur die Renten der gesetzlichen Rentenversicherung, nicht die durchschnittlich höheren Pensionen der Beamten, Richter und Soldaten. Obendrein werden oft nur die relativ niedrigen Renten einer Kunstfigur der OECD-Statistik erfasst, nämlich die des Average Production Worker. Betriebliche Alterssicherungsleistungen bleiben ebenfalls ausgeklammert. Und bei der Schätzung eines Äquivalents für die Mindestrente, die es in Deutschland erst seit der 2001 eingeführten Grundsicherung im Alter und bei Erwerbsminderung gibt, wird überdies nur ein Teil der Sozialhilfe bedacht, nämlich der Regelsatz. Damit wird auch Deutschlands Mindestrente unterschätzt.

Scruggs' Gesamtindikator der sozialpolitischen Generosität hat allerdings den Vorteil einer drei Jahrzehnte umfassenden Längsschnittbeobachtung. Aus der Vielzahl der Ergebnisse sind für Deutschlands Position zwei Resultate besonders berichtenswert:

Erstens: Im internationalen Vergleich liegt die Generosität der Sozialleistungen in Deutschland im gesamten Untersuchungszeitraum erneut nahe am Mittelwert aller 18 Länder (s. Tab. 1 im Anhang).

Zweitens: Scruggs' Daten zeugen auch von Einschnitten in die Sozialsysteme. Größere Einschnitte kamen in zwei Ländergruppen zustande. Die erste ist die der „sozialdemokratischen Wohlfahrtsstaaten“, zu denen Scruggs die nordeuropäischen Länder und die Niederlande zählt. Diese waren „,considerably less generous in 2002 than they were in the mid 1980s،45 - mit Ausnahme von Norwegen, das vom Nordseeöl profitierte. Deutschland gehört zu einer zweiten Ländergruppe mit Einschnitten in die Sozialleistungen, insbesondere durch Kürzungen bei Leistungen der Arbeitslosenversicherung: „Germany, France, and, to

keitsprüfung von Sozialleistungen und Sozialleistungen auf subnationaler Ebene angemessener berücksichtigt.

45 Scruggs, L.: Welfare state generosity, a.a.O. 
a lesser extent, Belgium, also appeared to be substantially less generous in 2002 than they were in the mid 1980s". 46

Allerdings sind zwei Relativierungen angebracht:

Erstens nahm die Generosität der Sozialprogramme in Deutschland nicht dramatisch ab, sondern moderat: Bei den Renten sank etwa der jahresdurchschnittliche Generositäts-Index von 7,8 im Zeitraum von 1971 bis 1990 auf 7,7 (1991-2002), bei der Arbeitslosenversicherung von 8,0 auf 7,6, und beim Krankengeld von 13,0 auf 12,9. Der Gesamtindex der Generosität ging von 28,8 auf 28,3 zurück. Im Lichte solcher Veränderungen spricht einiges für Paul Piersons Interpretation der Generositäts-Indizes: Die Zahlen zeigten eine bemerkenswerte Stabilität bei den Kernsystemen der sozialen Sicherung an - ,a pretty striking story of stability at the level of core programs"

Hinzu tritt - zweitens - ein auffälliger Kontrast ${ }^{48}$ : Deutschland, Frankreich und Belgien wiesen trotz Einschnitten in Sozialleistungen im Jahr 2002 höhere Sozialleistungsquoten als zuvor aus. Offensichtlich wurde die abnehmende Generosität der Sozialleistungen in einzelnen Sozialprogrammen von höheren Sozialausgaben an anderer Stelle begleitet. Dieser Kontrast bedarf einer Erklärung, die sich weder bei Scruggs noch in anderen Beiträgen zur vergleichenden Sozialpolitikforschung findet.

Ein Versuch zur Schließung dieser Lücke sei hier am Fall Deutschland gewagt. In der deutschen Sozialpolitik besteht tatsächlich eine auffällige Lücke zwischen einem sehr großen Sozialstaatsinput - gemessen an den Brutto- und den Nettosozialleistungsquoten - und einer oft nur mittelgradigen „Generosität“ der Sozialleistungen pro Empfänger in den Kernsystemen der sozialen Sicherung. Von mittelgradigen Leistungen zeugen nicht nur die Indikatoren der Dekommodifizierung und der sozialpolitischen „Generosität“, sondern auch etwa die einschlägigen, den Forschungsstand präsentierenden Kapitel im Oxford Handbook of the Welfare State. ${ }^{49}$

46 Ebd.

47 Pierson, P.: The Welfare State over the Very Long Run, Bremen: Zentrum für Sozialpolitik, ZESArbeitspapier 02/2011, 2011, 16.

48 Scruggs, L.: Welfare state generosity, a.a.O., 160.

49 S. die Beiträge in Castles, F.G. et al., a.a.O.: Bahle, T./Pfeifer, M./Wendt, C.: Social Assistance, 448461; Bradshaw, J./Finch, N.: Family Benefits and Services, 462-478; Fahey, T./Norris, M.: Housing 479-493; Freeman, R./Rothgang, H.: Health, 367-377; Hinrichs, K./Lynch, J.F.: Old-Age Pensions, 353366; Kangas, O.: Work Accident and Sickness Benefits, 391-405; Kenworthy, L.: Labour Market Activation, 435-447; Obinger, H./Wagschal, U.: Social Expenditure and Revenues, 333-352; Österle, 
Um die Ursachen der Lücke zwischen sehr hohen Sozialausgaben und mittelgradiger „Generosität“ zweifelsfrei zu klären, bedürfte es der Überprüfung von noch zu erhebenden international vergleichbaren Daten. Die verfügbaren Daten stützen allerdings die These, dass die Input-Output-Lücke der deutschen Sozialpolitik aus eigentümlichen Bedarfs- und Kostenstrukturen und speziellen parteipolitischen Konstellationen resultiert.

Erstens: Die Alterung der Bevölkerung in Deutschland ist weit vorangeschritten. Neben Italien und Japan verfügt Deutschland über die derzeit älteste Bevölkerung weltweit. Allein dadurch ist die Sozialstaatsklientel in Deutschland besonders groß - und wird weiter wachsen und erheblichen Bedarf nicht nur in den Alterssicherungssystemen, sondern auch in der Kranken- und der Pflegeversicherung sowie in den Mindestsicherungssystemen schaffen.

Zweitens: Deutschlands Wirtschafts- und Sozialpolitik laboriert an einer über mehrere Jahrzehnte vergleichsweise hohen Arbeitslosenquote, die die Nachfrage nach Sozialleistungen in die Höhe trieb und zu einer Ausweitung der Sozialstaatsklientel beitrug.

Drittens umfasst Deutschlands Sozialpolitik eine Reihe von leistungsstarken, kostspieligen Programmen jenseits der Renten-, Kranken- und Arbeitslosenversicherung. $\mathrm{Zu}$ diesen Programmen zählen die Pflegeversicherung, die aufgrund der voranschreitenden Alterung expandiert, sowie die weit ausgebauten Mindestsicherungssysteme, deren Adressatenkreise nicht zuletzt infolge von wirtschaftlichen Folgeproblemen der Wiedervereinigung wuchs - und vermutlich weiter wachsen wird, nicht zuletzt infolge einer langfristig voraussichtlich zunehmenden Altersarmut.

Viertens definieren die Mindestsicherungssysteme faktisch einen relativ hohen Mindestlohn. Dieser trägt zu hohen Lohnkosten bei und verstärkt jenen Kostendruck, der als „Baumols Krankheit“ bekannt wurde. ${ }^{50}$

Fünftens zählt ein weit ausgebautes, finanziell überdurchschnittlich aufwendiges Gesundheitswesen zur Sozialpolitik der Bundesrepublik. Das Gesundheitssystem

A./Rothgang, H.: Long-Term Care, 378-390; Priestley, M.: Disability, 406-419; Sjöberg, O./Palme, J./Carroll, E.: Unemployment Insurance, 420-434, und Stephens, J.D.: The Social Rights of Citizenship, 511-525.

50 Der Kostendruck entsteht, wenn Sektoren mit geringerer Produktivität (z.B. weite Bereiche des öffentlichen Sektors) ihre Lohnpolitik weitgehend an der Lohnentwicklung produktivitätsstärkerer Sektoren (insbesondere der Industrie) orientieren (Baumol, W.J.: Health Care, Education and the Cost Disease: A Looming Crisis for Public Choice, in: Public Choice, 77/1, 1993, 17-28). 
gewährt im Grundsatz jedem Versicherten den gleichen Anspruch auf Gesundheitsdienstleistungen, unabhängig von der Höhe des Versicherungsbeitrags eine Systementscheidung, die beim internationalen Vergleich zu wenig beachtet wird.

Sechstens tritt die Wirkung von Kurswechseln in der Sozialstaatsfinanzierung hinzu, vornehmlich hin zu einer auf Beitragssatzstabilität bedachten Politik. Am klarsten sichtbar ist diese Richtungsänderung im Bereich der Alterssicherung. Diese vollzog insbesondere unter der rot-grünen Regierung Schröder (19982005) den Übergang von der rentenniveau- zur beitragssatzorientierten Rentenpolitik. Dieser Richtungswechsel strahlt auch auf andere Sozialpolitikfelder aus und sorgt für hohen Kostendruck und tendenziell sinkende Lohnersatzraten insbesondere bei jenen Sozialleistungen, die, wie die Rente und die Arbeitslosenversicherung, größtenteils aus Beiträgen finanziert werden.

Siebtens schließlich spiegeln die Größe und die Vielschichtigkeit des von der deutschen Sozialpolitik geschützten Personenkreises eine besonders sozialstaatsfreundliche Parteienlandschaft wider. ${ }^{51}$ Deutschlands Sozialpolitik wird nicht nur von einer Sozialstaatspartei gefördert und dabei kaum von einer marktliberalen Partei nach Art der britischen Conservative Party gebremst. In Deutschland sind es vielmehr zwei große Sozialstaatsparteien, die für die Sache der Sozialpolitik eintreten: die SPD und die CDU/CSU. Entsprechend groß - im internationalen Vergleich sogar überdimensioniert - ist die Größe der prinzipiell sozialstaatsfreundlichen Wählerschaft. Seit der deutschen Einheit haben es die beiden großen Sozialstaatsparteien obendrein mit der Partei „Die Linke“ als einer radikalen Befürworterin des Wohlfahrtsstaates zu tun. All diese Parteien streben danach, alle Gruppen und alle Sozialrisiken ihrer Wähler und der von ihnen umworbenen Zielgruppen mit sozialpolitischen Programmen zu versorgen - mit dem Kalkül, hierdurch Loyalität und Wählerstimmen zu halten oder zu erwerben. Auch deshalb ist die Reichweite der Sozialpolitik so groß, ihre Architektur so vielschichtig wie unter II. beschrieben und ihre Sozialstaatsklientel von einer Größenordnung, die rund 40 Prozent der Wahlberechtigten entspricht. ${ }^{52}$ Entsprechend hoch

51 Schmidt, M.G.: Sozialpolitik in Deutschland. Historische Entwicklung und internationaler Vergleich, Wiesbaden, 3. Aufl. 2005; ders. (Hg.): Bundesrepublik Deutschland 1982-1989. Finanzielle Konsolidierung und institutionelle Reform, Baden-Baden. Geschichte der Sozialpolitik in Deutschland seit 1945, Bd. 7, Baden-Baden, 2005.

52 „Sozialstaatsklientel“ meint den Teil der Wahlberechtigten, der seinen Lebensunterhalt überwiegend aus Sozialeinkommen finanziert. Die Schätzung basiert auf der Auswertung der einschlägigen Daten in Statistisches Bundesamt (Hg.): Statistisches Jahrbuch 2011. Für die Bundesrepublik Deutschland mit »Internationalen Übersichten«, Wiesbaden, 2011. Der größte Teil entfällt auf Rentner und Pensionäre. Mit 
ist der Bedarf an Sozialleistungen - und entsprechend ausgeprägt der finanzielle Aufwand zur Bedarfsdeckung.

\section{Sozialpolitische Regulierungen der Arbeitswelt}

Deutschlands Sozialpolitik besteht nicht nur aus den Systemen der sozialen Sicherung. Sie reguliert auch die Arbeitswelt durch Arbeitsschutz, Arbeitsmarktpolitik, Beschäftigungsschutz und Mitbestimmung. ${ }^{53}$ Während sich die Datengrundlage für den internationalen Vergleich sozialer Sicherungssysteme als durchaus beträchtlich erweist, sind vergleichbare Daten für die Regulierung der Arbeitswelt nur spärlich vorhanden. Eher wohldefiniert sind noch die Daten zur Arbeitslosenversicherung, zur Arbeitsmarktpolitik und inzwischen auch zum Beschäftigungsschutz. Das ist ein Verdienst vor allem der OECD, die hierzu umfangreiche Messungen der sozialpolitischen Protektion von Beschäftigungsverhältnissen seit den 1980er Jahren vorgelegt hat (employment protection legislation). ${ }^{54}$

Die Auswertung dieses Zahlenwerkes fördert sechs besonders berichtenswerte Befunde zu Deutschlands Position im internationalen Vergleich zutage:

- Deutschland zählt seit den ersten Messungen in den 1980er Jahren im OECD-Vergleich bis heute zu den Ländern mit einem insgesamt überdurchschnittlich hohen Beschäftigungsschutz.

- Der Beschäftigungsschutz ist in Deutschland erheblich größer als in der englischsprachigen Länderfamilie. Übertroffen wurde er in den späten 1980er Jahren vor allem von den südeuropäischen Ländern und Schweden, ${ }^{55}$ sowie heutzutage von den südeuropäischen Ländern, Frankreich, Mexiko und der Türkei. ${ }^{56}$

- Deutschlands Arbeitsmarkt-Insider - die unbefristet Beschäftigten - sind besonders privilegiert. Unbefristete Beschäftigungsverhältnisse werden in Deutschland so stark geschützt wie in Schweden. Dies wird im OECDLänder-Vergleich nur noch vom portugiesischen und tschechischen Ar-

Abstand folgen die Empfänger von Leistungen aus den Mindestsicherungssystemen und die Bezieher von Arbeitslosengeld - um nur die allerwichtigsten Gruppen zu nennen.

53 Vgl. hierzu die international vergleichenden Übersichten bei Dittrich, W., a.a.O.; Hörisch, F., a.a.O.

54 "Employment protection" umfasst die Regulierung der Einstellung und der Kündigung von Arbeitskräften. Die wichtigsten Daten finden sich in: $O E C D$, Employment Protection, a.a.O., 66; Venn, D., a.a.O.; www.oecd.org/employment/key_statistics - Tabelle Employment Protection, Abruf am 9.1.2012.

55 OECD, Employment Protection, a.a.O., 66.

56 Datenstand: 2008, Venn, D., a.a.O., 8. 
beitsmarktrecht übertroffen. ${ }^{57}$ Der Schutz für befristete Jobs hingegen - auf sie entfallen in Deutschland derzeit rund 15 Prozent der abhängigen Beschäftigung $^{58}$ - ist inzwischen mit 1,3 Punkten auf einer von 0 bis 6 reichenden Skala auch international als niedrig einzustufen (Stand: 2008). Unterboten wird er nur von dem noch geringeren Beschäftigungsschutz in den englischsprachigen Ländern, in Schweden, der Schweiz, den Niederlanden, Japan und Israel.

- Die Privilegierung der deutschen Arbeitsmarkt-Insider ragt auch im internationalen Vergleich hervor - insbesondere im Spiegel der Entwicklung seit den 1990er Jahren. Die Differenz zwischen dem Beschäftigungsschutz für unbefristete und für befristete Jobs in Deutschland beträgt auf der von 0 bis 6 reichenden Skala der employment protection im Jahre 2008 1,7 Punkte (3,0 minus 1,3). Diese Differenz wird im internationalen Vergleich nur noch von der Tschechischen Republik, der Slowakei, Portugal, Slowenien und Schweden überboten. ${ }^{59}$

- Die Liberalisierung des Arbeitsmarktes, die in den 1980er Jahren in den OECD-Mitgliedstaaten begann, ging an Deutschland nicht vorbei. ${ }^{60}$ Der Beschäftigungsschutz wurde insgesamt gelockert, wie in nahezu allen anderen OECD-Mitgliedstaaten, soweit diese nicht bereits über liberalisierte Arbeitsmärkte verfügten, wie die englischsprachige Ländergruppe. Gemessen am ursprünglichen Niveau und am Tempo der Liberalisierung hält Deutschland auch beim Rückbau des Beschäftigungsschutzes eine Position ungefähr in der Mitte der OECD-Mitgliedstaaten. Deutschland folgt nicht nur in weiten Teilen seiner Wirtschafts- und Sozialpolitik einem Mittelweg, sondern auch bei der Liberalisierung seiner Arbeitsmärkte. ${ }^{61}$

- Trotz Arbeitsmarktliberalisierung behalten die deutschen ArbeitsmarktInsider ihre relative Privilegierung. Der Beschäftigungsschutz für die unbefristet Beschäftigten wurde durch die Liberalisierungspolitik der letzten zwei bis drei Jahrzehnte weit weniger vermindert als der Schutz befristeter Arbeitsverhältnisse. Der Beschäftigungsschutz für unbefristete Jobs bleibt bis heute auf einem international überdurchschnittlich hohen Niveau. ${ }^{62}$ Der Be-

57 Datenstand: 2008, www.oecd.org/employment/key_statistics, Abruf am 9.1.2012.

58 Datenstand: 2010, www.oecd.org/employment/key_statistics, Tabelle Temporary and part-time employment, Abruf am 9.1.2012.

59 Berechnet auf der Basis von www.oecd.org/employment/key_statistics: Tabelle Employment Protection, Abruf am 9.1.2012. Siehe Tabelle 1 im Anhang.

60 Venn, D., a.a.O., 8 f.; Höpner, M. et al., a.a.O.; Siegel, N.A., a.a.O.

61 Schmidt, M.G.: Das politische System Deutschlands. Institutionen, Willensbildung und Politikfelder, 2. Aufl. München, 2011, 444-464; ders.: Der mittlere Weg der Liberalisierungspolitik? Deutschland im internationalen Vergleich, in: Jesse, E./Sturm, R. (Hg.): „Superwahljahr“ 2011 und die Folgen, Wiesbaden 2012, 285-305, so auch Höpner et al., a.a.O., 23.

62 Siegel, N.A., a.a.O.; Venn, D., a.a.O., 8 f. 
schäftigungsschutz für befristete Stellen hingegen wurde in Deutschland überdurchschnittlich stark abgesenkt: Er schrumpfte schon von den 1980er bis in die 1990er Jahre ${ }^{63}$, und er sank allein von 1998 bis 2008 abermals auf der von 0 bis 6 reichenden Skala von 2,0 auf 1,3. Nur sechs andere OECDMitgliedstaaten erreichten oder übertrafen den deutschen Liberalisierungsschub im Bereich der befristeten Beschäftigungsverhältnisse. ${ }^{64}$

Die Daten zu Niveau, Wandel und Beharrungsvermögen des Beschäftigungsschutzes stützen am ehesten jene Kritiker, die die deutsche Sozialpolitik der Mittäterschaft bei der Gesellschaftsspaltung und beim Abbau von Arbeitnehmerschutzrechten bezichtigen und ihr vorhalten, sie kümmere sich hauptsächlich um unbefristete und vollzeitbeschäftigte Arbeitnehmer des primären Arbeitsmarktes. $^{65}$

Doch bei aller Kritik an der Privilegierung der Arbeitsmarkt-Insider in Deutschland sind auch die Beschäftigungseffekte flexiblerer Arbeitsmärkte zu bedenken. Der Rückbau des Beschäftigungsschutzes für befristete Arbeitsverhältnisse seit den 1980er Jahren verwandelte den hochgradig inflexiblen deutschen Arbeitsmarkt in einen Arbeitsmarkt mit erheblich höherer Beschäftigungsdynamik zunächst vor allem bei zeitlich befristeten Tätigkeiten, der Teilzeitarbeit und der geringfügigen Beschäftigung, den abgabenrechtlich privilegierten „400 EuroJobs“, später auch bei der sozialversicherungspflichtigen Beschäftigung.

Die gesetzlichen Grundlagen der Arbeitsmarktflexibilisierung wurden in den 1980er Jahren zunächst noch tastend gelegt und sodann in großen Schritten durch die Arbeitsmarktreformen der rot-grünen Regierung Schröder weitergeführt. Den beschäftigungspolitischen Ertrag dieser Reformen erntete allerdings nicht mehr die rot-grüne Koalition, sondern ihre Nachfolgerin, die von 2005 bis 2009 amtierende zweite Große Koalition, und ab 2009 die schwarz-gelbe Koalition aus $\mathrm{CDU} / \mathrm{CSU}$ und FDP.

63 OECD, Employment Protection, a.a.O., 66.

64 Es sind dies die Niederlande und Schweden sowie - allerdings von einem sehr hohen Ausgangsniveau startend - Griechenland, Italien, Portugal und die Slowakei, www.oecd.org/employment/key statistics, Tabelle Employment protection. Zum Vergleich mit den 1980er Jahren siehe OECD, Employment Protection, a.a.O., 66.

65 Vgl. Lessenich, S.: Die Neuerfindung des Sozialen. Der Sozialstaat im flexiblen Kapitalismus, Frankfurt a.M.-New York, 2009 sowie die - für die Sozialpolitik insgesamt nicht überzeugende - These, dass Deutschlands Sozialstaatsparteien zu einem liberal-kommunitaristischen Sozialstaatsmodell konvertierten (Seeleib-Kayser, M./van Dyk, S./Roggenkamp, M., a.a.O.). 


\section{Erwerbsbeteiligung, Beschäftigung und Arbeitslosigkeit}

Zur Kritik an Deutschlands Sozialpolitik gehört die These, dass sie - trotz ihres Strebens nach „Arbeitsförderung“ - für eine unterdurchschnittliche Erwerbsbeteiligung mitverantwortlich sei. Die Lehre von den „Drei Welten des Wohlfahrtsstaates ${ }^{\text {“66 }}$ etwa sieht hierfür zwei Hauptursachen: eine Arbeitsteilung zwischen Markt und Staat, die den Staat und die Beschäftigung im Staatssektor in Deutschland als „,konservativem Wohlfahrtsstaat“ am kürzeren Zügel führe als in Nordeuropa, und die Vorherrschaft des männlichen Brotverdienermodells in der Familie, die mit unbezahlter Hausarbeit von Frauen und einer geringeren Frauenerwerbsbeteiligung einhergehe. Weil außerdem die Hochlohnpolitik der von den männlichen Erwerbstätigen im Industriesektor beherrschten Gewerkschaften hinzukomme, so manche Vertreter dieser Lehre, sei ein weiterer Anreiz zur arbeitssparenden Investition und entsprechend geringerer Erwerbsbeteiligung gegeben. Obendrein seien die politisch-institutionellen Rahmenbedingungen für einen erfolgreich ,aktivierenden Wohlfahrtsstaat" in Deutschland schwächer als in unitarischen Staaten wie Dänemark oder Großbritannien. ${ }^{67}$

Allerdings hält diese Kritik einer Betrachtung der Arbeitsmarktdaten seit den 1990er Jahren nicht stand.

Die Erwerbsbeteiligung der Personen im Alter von 15-64 Jahren ist in Deutschland seit mehr als einem Jahrzehnt - auch im Vergleich mit allen anderen OECD-Ländern - überdurchschnittlich hoch und der Tendenz nach im Steigen begriffen. Das gilt sowohl für die Erwerbsbeteiligung der Männer als auch für die der Frauen. ${ }^{68}$

Eindrücklich entwickelten sich auch die Beschäftigungsquoten bei Männern wie Frauen. ${ }^{69}$ Sie fallen in Deutschland ebenfalls überdurchschnittlich hoch aus und entwickeln sich weiter positiv und über dem OECD-Durchschnitt - zunächst nur bei den Frauen im erwerbsfähigen Alter, seit 2008 auch bei den Männern.

Ein bemerkenswerter Wandel betrifft die Erwerbsquote von Frauen. Sie entwickelte sich bis in die 1980er Jahre nur durchschnittlich oder gar unterdurch-

66 Esping-Andersen, G.: Three Worlds, a.a.O.

67 Dingeldey, I.: Der aktivierende Wohlfahrtsstaat. Governance der Arbeitsmarktpolitik in Dänemark, Großbritannien und Deutschland, Frankfurt a.M., 2011.

68 OECD: OECD Employment Outlook 2011, Paris, 2011, 240-242.

69 Im Sinne der employment-population ratios, OECD: Employment Outlook 2011, a.a.O., 239-241. 
schnittlich. ${ }^{70}$ Inzwischen ist sie jedoch deutlich gestiegen, wodurch der Abstand zu den hier führenden nordischen Staaten schrumpfte: Der Unterschied zwischen den deutschen und schwedischen Frauenerwerbsquoten (in Prozent der weiblichen Bevölkerung im Alter von 15 bis 64), der noch 198925 Prozentpunkte betrug, verkürzte sich bis 2010 auf 6 Prozentpunkte. ${ }^{71}$

Der internationale Vergleich der Arbeitsmarktdaten deckt allerdings auch eine besondere Schwäche der deutschen Sozial-und Wirtschaftspolitik seit den 1980er Jahren auf: die Arbeitslosigkeit. Eine im OECD-Ländervergleich überdurchschnittlich hohe Arbeitslosenquote kennzeichnete den deutschen Arbeitsmarkt erstmals 1985, anschließend von 1987 bis 1989 und nach dem Ende des Einheitsbooms erneut von 1994 bis 2008. Auch wenn die Daten seit 2009 zum ersten Mal seit längerem wieder auf eine unterdurchschnittliche Arbeitslosenquote in Deutschland verweisen, spricht der Gesamtbefund der vergangenen drei Jahrzehnte doch für die These, dass die Achillesferse der deutschen Sozial- und Wirtschaftspolitik in der Arbeitslosigkeit zu suchen ist. ${ }^{72}$

Ergänzt wird diese These durch den hohen Anteil der Langzeitarbeitslosen an der Gesamtarbeitslosigkeit. ${ }^{73}$ Auch bei den Langzeitarbeitslosen liegt Deutschland über dem OECD-Durchschnitt. So belief sich der Anteil der mindestens sechs Monate lang Arbeitslosen in Deutschland 2010 auf 63,5 Prozent im Vergleich zu 48,5 Prozent im gewichteten Durchschnitt der OECD-Länder. Für Personen, die mindestens zwölf Monate arbeitslos waren, erreichte dieser Wert 47,4 Prozent gegenüber durchschnittlich 32,4 Prozent in der OECD-Welt.

Die Auswertung der Arbeitsmarktdaten deckt ein weiteres bemerkenswertes Muster auf: In nur wenigen anderen Ländern korreliert das Arbeitslosigkeitsrisiko so stark mit dem Qualifikationsniveau wie in Deutschland. So waren von den überdurchschnittlich ausgebildeten 15-64-jährigen Deutschen im Jahre 2009 nur 3,5 Prozent arbeitslos, unter den unterdurchschnittlich Qualifizierten aber waren es 17,0 Prozent. Nur in drei OECD-Mitgliedstaaten ist die Spreizung zwischen diesen beiden Qualifikationsgruppen noch größer als hierzulande: in der Tsche-

70 Schmidt, M.G.: Erwerbsbeteiligung von Frauen und Männern im Industrieländervergleich, Opladen, 1993.

71 Auswertung auf der Basis von OECD: Historical Statistics 1960-1990, Paris, 1992, 39 und OECD: Employment Outlook 2011, a.a.O., 241.

72 OECD: Employment Outlook 2011, a.a.O., 238; für frühere Daten $O E C D$ : Historical Statistics, a.a.O., 43, 45 und $O E C D$ : Employment Outlook, Paris, 2007.

73 OECD: Employment Outlook 2011, a.a.O., 259. 
chischen Republik, der Slowakei und in Ungarn. ${ }^{74}$ Erheblich geringer ist sie in den englischsprachigen Ländern, in den südeuropäischen Staaten und in Frankreich. $^{75}$

Die Persistenz der Arbeitslosigkeit, der anhaltend hohe Anteil der Langzeitarbeitslosen, die sehr hohen Arbeitslosigkeitsrisiken von unterdurchschnittlich Qualifizierten und die im vorangehenden Kapitel erörterte Privilegierung der Arbeitsmarkt-Insider gehören zu den Schwächen der sozialpolitischen Regulierung der Arbeitswelt in Deutschland. Sie sind allerdings mit dem Landgewinn bei den Beschäftigungs- und den Erwerbsquoten zu verrechnen: Diese erweisen sich als Zeichen einer höheren Beschäftigungsdynamik der deutschen Sozialund Wirtschaftspolitik.

\section{Sozialpolitik, gesellschaftliche Ungleichheit und Armuts- bekämpfung}

Die „Suche nach Sicherheit“, so der Titel und die Hauptthese von Eckhard Conzes Geschichte der Bundesrepublik Deutschland seit 1949, ${ }^{76}$ treibt Deutschlands Bürger und die sie regierenden Politiker um. Der Sozialpolitikforschung leuchtet diese These in besonderem Maße ein. Denn die Sicherung gegen Risiken, gegen die sich der Einzelne meist nicht zureichend schützen kann, ist eines der Hauptanliegen der deutschen Sozialpolitik. Sicherheit buchstabiert sie als ausgeprägten Sozialschutz insbesondere für Beamte, Angestellte, Arbeiter und ihre nichterwerbstätigen Familienmitglieder - im Bereich der Renten- und der Arbeitslosenversicherung in dosierter Abhängigkeit von Beitragssatz und Beitragsdauer sowie untermauert von leistungsfähigen Mindestsicherungssystemen für Bedürftige. Dieses Leitbild unterscheidet die Sozialpolitik in Deutschland von anderen Ländern: von der primären Armutsbekämpfung, die stilprägend für die britische Sozialpolitik wurde, von der Egalisierung, nach der insbesondere sozialdemokratisch dominierte Regierungen streben, und von der Betonung der geburtenorientierten Bevölkerungs- und Familienpolitik Frankreichs. ${ }^{77}$

74 Ebd., $251 \mathrm{ff}$.

75 Ebd.

76 Conze, E.: Die Suche nach Sicherheit: Eine Geschichte der Bundesrepublik Deutschland von 1949 bis in die Gegenwart, Berlin, 2009.

77 Kaufmann, F.-X.: Varianten des Wohlfahrtsstaats. Der deutsche Sozialstaat im internationalen Vergleich, Frankfurt a.M., 2003. 
Der Vorrang für Sicherheit und beitragsbezogene Leistungen resultiert in Sozialleistungen unterschiedlicher Höhe: Wer länger und mit höheren Beiträgen versichert war, erhält etwa eine tendenziell höhere Rente als derjenige, der nur kürzere Beitragszeiten und niedrigere Beitragssätze nachweisen kann. Diese Schichtung der Sozialeinkommen nimmt zu, wenn die Zahl und der Anteil befristeter Arbeitsverhältnisse zunehmen und die Erwerbskarrieren unstetiger werden als die des durchweg vollzeitbeschäftigten Arbeitnehmers.

Spuren dieser Spreizung finden sich im internationalen Vergleich wieder. Wird die Sozialpolitik etwa an der Nettolohnersatzrate der Sozialleistungen gemessen, kommen unterschiedliche Ergebnisse zustande. Großzügig ist das Krankengeld, das in Deutschland im Krankheitsfall die Lohnfortzahlung eines Großteils des Arbeitsentgelts vorsieht. Je nach Indikator und Quelle erweisen sich die Lohnersatzraten der deutschen Arbeitslosenversicherung im Vergleich der OECDMitgliedstaaten als überdurchschnittlich oder durchschnittlich. Weit auseinander liegen die Schätzungen der Lohnersatzleistung in der Alterssicherung: Je nach Quelle und Indikator befinden sich die Lohnersatzraten der deutschen gesetzlichen Rentenversicherung über, auf oder unter dem Durchschnitt der OECDMitgliedstaaten. Beim Vergleich von Sozialhilfeleistungen hingegen erzielt Deutschland meist durchschnittliche Lohnersatzraten. ${ }^{78}$ Gemessen an den über alle Sozialversicherungszweige gemittelten Lohnersatzraten schließlich ist die deutsche Sozialpolitik beim internationalen Vergleich erneut in der Mitte platziert - mitunter auch über dem Durchschnitt. ${ }^{79}$ Und im Spiegel der Generosität der Sozialleistungen rangiert Deutschland meist hinter den skandinavischen Ländern und in der Regel vor den USA und anderen englischsprachigen Staaten.

Der Abbau sozialer Ungleichheit ist nicht das Hauptanliegen des deutschen Sozialstaats, sondern ein dem Sicherheitsstreben nachgeordnetes Ziel. Insoweit ist es nicht allzu überraschend, dass die deutsche Sozialpolitik im internationalen Vergleich durchschnittlicher Lohnersatzraten nicht an der Spitze liegt. Gleiches gilt für Ungleichheits- und Armutsindizes. Davon zeugte unlängst eine Studie der Bertelsmann-Stiftung zur sozialen Gerechtigkeit. ${ }^{80}$

78 Vgl. nur die Beiträge zu Castles, F.G. et al., a.a.O.; ferner Starke, P./Obinger, H./Castles F.G.: Convergence to where: in what ways, if any, are welfare states becoming similar?, in: Journal of European Public Policy, 15/4, 2008, 975-1000; Scruggs, L./Allan, J.: Welfare-state decommodification, a.a.O.; Wenzelburger, G./Zohlnhöfer, R./Wolf, F., a.a.O.

79 Scruggs, L.: The generosity of social insurance, a.a.O.

80 Vgl. Bertelsmann-Stiftung: Soziale Gerechtigkeit in der OECD - Wo steht Deutschland? Sustainable governance Indicators 2011, Gütersloh, 2011. Bei der Armutsvermeidung nahm Deutschland laut dieser Studie den 14. Rang unter 31 Ländern ein, bei der Arbeitsmarkt-Dimension den Rang 15, bei der 
Vorrang für Sicherheit heißt allerdings nicht Verzicht auf sozialen Ausgleich. Ihn gibt es in der gesamten deutschen Sozialpolitik. Und obwohl die Bekämpfung von Ungleichheit nicht ihr Hauptziel darstellt, wirkt die deutsche Sozialpolitik beim Abbau sozialer Ungleichheit mit, und zwar mit überraschend hohem Erfolg. Davon zeugte der 3. Reichtums- und Armutsbericht der Bundesregierung von 2005: Ihm zufolge wurde die Armutsquote in Deutschland allein durch Sozialtransfers wie Arbeitslosengeld II, Kindergeld, Kinderzuschlag, Wohngeld und das frühere Erziehungsgeld im Jahr 2005 halbiert, von 26 Prozent der Bevölkerung auf 13 Prozent. Das ist ein Ergebnis der Armutsbekämpfung, das sich auch im Vergleich mit allen Mitgliedstaaten der Europäischen Union sehen lassen kann. ${ }^{81}$ Diese Befunde widersprechen der Meinung, die deutsche Sozialpolitik verteile nur wenig zwischen Arm und Reich um. Deutschlands Sozialpolitik bestätigt vielmehr einen Befund internationaler Vergleiche: Je höher der Anteil der öffentlichen Sozialausgaben am Sozialprodukt, desto stärker wird die markterzeugte Einkommensungleichheit durch Sozialtransfers eingedämmt. Deutschlands überdurchschnittlich großer Sozialetat ist Teil eines Mechanismus, der die hohe markterzeugte Einkommensungleichheit im Lande ${ }^{82}$ in überdurchschnittlichem Maße vermindert.

Hinzu kommt die Umverteilung durch die Steuerpolitik. Steuern im Verein mit Sozialtransfers verringern nicht nur das Armutsrisiko in großem Maße, sondern auch die überdurchschnittlich hohe Einkommensungleichheit. Dem Oxford Handbook of Economic Inequality zufolge reduzieren Deutschlands Steuern und Sozialtransfers die Einkommensungleichheit fast so stark wie in den diesbezüglich führenden skandinavischen Ländern und beinahe doppelt so stark wie in den USA. $^{83}$ Noch auffälliger ist der Abbau von Einkommensungleichheit und Armutsrisiken durch Sozialtransfers und Steuern laut neuesten OECD-Daten: Ihnen

Gleichheit ebenfalls den Rang 15, bei der Generationengerechtigkeit den 11. Rang und beim Zugang zu Bildung den 22. Rang. Im Gesamtergebnis lag Deutschland auf dem Rang 15 der Gesamtskala im OECD-Mitgliedstaaten-Vergleich.

81 Lebenslagen in Deutschland. Der 3. Armuts- und Reichtumsbericht der Bundesregierung, Berlin, 2005, XII und 328. Die Armutsgrenze lag bei 60 Prozent des mittleren Äquivalenzeinkommens. Die Daten finden sich in Tabelle 1 im Anhang.

82 Die Einkommen in Deutschland sind - gemessen an der Primärverteilung - überdurchschnittlich ungleich verteilt, ähnlich ungleich wie in den USA (Brandolini, A./Smeeding, T.M.: Income Inequality in Richer and OECD Countries, in: Salverda, W./Nolan, B./Smeeding, T.M. (Hg.): The Oxford Handbook of Economic Inequality, Oxford, 2009, 71-99, hier 93). So auch ein Befund der Income Distribution Database der OECD. Vgl. den Datenauszug für 2009 in OECD: OECD Economic Surveys: Chile, Paris, 2012, 12.

83 Vgl. Brandolini/Smeeding, a.a.O., 92 ff., vgl. Tabelle 1 im Anhang. 
zufolge liegt Deutschland beim Abbau der Einkommensungleichheit zusammen mit Belgien, Finnland und Österreich in der Spitzengruppe der OECD-Länderund bei der Verminderung des Armutsrisikos auf dem zweiten Platz hinter Frankreich und vor den skandinavischen Ländern. ${ }^{84}$

Auch diese Daten rücken Deutschlands Sozialpolitik in ein - im Vergleich zu ihrer Kritik - vorteilhafteres Licht.

\section{Im „Reformstau“ gefangen oder doch reformfähig? Deutschlands Sozialpolitik vor neuen Herausforderungen}

Inwieweit kommt die Sozialpolitik in Deutschland mit neuen Herausforderungen zurecht - wie Europäisierung und Globalisierung einerseits, schwächerem Wirtschaftswachstum, höherer Arbeitslosigkeit, Engpässen bei der Sozialstaatsfinanzierung und Folgekosten der deutschen Einheit andererseits?

Anpassungen der Sozialpolitik an neue Herausforderungen sind überall eine politisch heikle Angelegenheit. Sie sind umso heikler, je mehr sie politisch meist unpopuläre Umbau- und Rückbaumaßnahmen erfordern. ${ }^{85}$ Einer weit verbreiteten Sichtweise zufolge tut sich die Sozialpolitik in Deutschland mit solchen Herausforderungen besonders schwer. Sie gilt als Teil eines politischen Systems, das sich aufgrund besonders vieler Vetospieler und des Wettbewerbs zwischen zwei großen Sozialstaatsparteien Reformträgheit, mitunter sogar „Reformstau“ nachsagen lassen muss. Zudem, so heißt es, bremsen die einflussreichen Sozialversicherungen die Reformfähigkeit, weil die Sozialbeiträge den versicherten Arbeitnehmern Eigentumsrechte verschaffen, die sich bei der finanziellen Konsolidierung der Sozialbudgets als Hindernisse erweisen. Überdies neige die deutsche Sozialpolitik zu pfadabhängigen Reformen und dazu, Reformen verspätet und in kleinen Schritten anzugehen. ${ }^{86}$

Tatsächlich reagierte die Sozialpolitik in Deutschland zunächst wenig sensibel auf die neuen Herausforderungen, die sich insbesondere seit der Mitte der 1970er Jahren häuften. Allein die „Trendwende“687 der Wirtschafts- und Sozialpolitik Mitte der 1970er Jahre hatte die Großwetterlage für die Sozialpolitik fundamen-

84 Datenstand: 2009. Die Daten entstammen der OECD Income Distribution Database, für Details Tabelle 1, Spalten 12, 13 und 14 im Anhang.

85 Pierson, $P$., a.a.O.

86 Ritter, G.A.: Der Preis der Einheit. Die Wiedervereinigung und die Krise des Sozialstaats, München, 2. Aufl. 2007.

87 Dahrendorf, R. (Hg.): Trendwende - Europas Wirtschaft in der Krise, München-Wien-Zürich, 1977. 
tal verändert: Nunmehr war ihr finanzieller Spielraum geschrumpft, ihr Aufgabenkreis aber gewachsen. Die Bundesregierung reagierte zunächst nur zögerlich auf die neue Lage. Noch beflügelte ein robuster Aufschwungs- und Machbarkeitsoptimismus die Sozialpolitiker in Regierung und Opposition zum fortwährenden Streben nach „Immer-Mehr“, „Immer-Besser““ und „Immer-Größer“"88. Noch gegen Ende des Jahres 1974, schon im Schatten einer schweren Wirtschaftskrise, unterstellten die Sozialpolitikplanungen im Bundesministerium für Arbeit und Sozialordnung für den nächsten Fünfjahreszeitraum ein durchschnittliches Wachstum der Bruttoverdienste der Arbeitnehmer in Höhe von 10,1 Prozent pro Jahr. ${ }^{89}$

Einen ersten, noch zwischen Einsparungen und Status-quo-Festschreibung schwankenden Anlauf zur Anpassung des Sozialstaats an die neuen Herausforderungen unternahm die SPD/FDP-Regierung unter Kanzler Helmut Schmidt. Der beherzte Einstieg in die sozialstaatlichen Anpassungsreformen erfolgte dann unter der ab Oktober 1982 regierenden Koalition aus CDU/CSU und FDP ${ }^{90}$ Mit dem Ziel, die Sozialfinanzen zu konsolidieren, kürzte die bürgerlich-liberale Koalition Sozialleistungen und erhöhte die Sozialbeiträge - ein typischer Fall von Reformen erster Ordnung, die hier mehr, dort weniger von dem Althergebrachten vorsehen, aber weder die Instrumente noch die Steuerungsphilosophie ändern. Später folgten Reformen zweiter Ordnung. Sie führten neue Steuerungsinstrumente ein, die hauptsächlich die großen Sozialversicherungen stabilisieren sollten, etwa den selbstregulierenden Finanzverbund zwischen Beitragszahlern, Rentnern und dem Bund in der 1989 beschlossenen „Rentenreform 1992”. Reformen dritter Ordnung, die zudem die Steuerungsphilosophie änderten, kamen unter der rot-grünen Regierung zustande. Doch allein die Konsolidierung der Sozialfinanzen bis Ende der 1980er Jahre hatte tiefe Spuren in der Staatstätigkeit hinterlassen. Der Anteil der öffentlichen Sozialausgaben am Bruttoinlandspro-

88 So die - in kritischer Distanz zu den Sozialpolitikern vorgetragenen - Äußerung des Bundesfinanzministers der Jahre von 1974 bis 1978, Hans Apel, bei der Vorlage des Bundeshaushaltes 1975 im September 1974, zitiert nach Hockerts, H.G./Süß, W.: Gesamtbetrachtung: Die sozialpolitische Bilanz der Reformära, in: Hockerts, H.G. (Hg.): Bundesrepublik Deutschland 1966-1974. Eine Zeit vielfältigen Aufbruchs. Geschichte der Sozialpolitik in Deutschland seit 1945, Bd. 5, Baden-Baden, 2007, 943-962, hier 955 .

89 Schmähl, W.: Sicherung bei Alter, Invalidität und für Hinterbliebene, in: Hockerts, H.G., a.a.0., 2007, 407-482, hier 481 .

90 Zur Sozialpolitik dieser Koalition Schmidt, M.G.: Bundesrepublik Deutschland 1982-1989, a.a.O., hg. v. BMAS/Bundesarchiv, Bd. 7, Baden-Baden, 2005; Ritter, Preis der Einheit, a.a.O.; Ritter, G.A. (Hg.): Bundesrepublik Deutschland 1989-1994. Sozialpolitik im Zeichen der Vereinigung. Geschichte der Sozialpolitik in Deutschland seit 1945, Bd. 11, Baden-Baden, 2007. 
dukt etwa schrumpfte von 33,0 Prozent 1982 auf 29,1 Prozent im Jahre $1990 .^{91}$ Das war zumindest in quantitativer Hinsicht ein Erfolg, wie der Sachverständigenrat zur Begutachtung der gesamtwirtschaftlichen Entwicklung in seinem Jahresgutachten 1986/87 rückblickend schrieb, wenngleich er die fehlende Nachhaltigkeit und die anhaltend hohen, tendenziell steigenden Sozialbeiträge bemängelte. $^{92}$

Nach anfänglichem Zögern und der Rücknahme von Reformen ihrer Vorgänger ergänzte die rot-grüne Koalition die Sozialstaatsanpassung ihrerseits mit tiefgreifenden Reformen. Nunmehr kamen auch Reformen dritter Ordnung zum Zuge Kursänderungen, die sowohl neue Instrumente als auch eine neue Regelungsphilosophie einführten. Zwei Paradigmenwechsel in der Rentenpolitik gaben den Auftakt. Der erste bestand aus der Intensivierung des - schon bei der Vorgängerregierung einsetzenden - Übergangs von der rentenniveauorientierten zur beitragssatz- oder einnahmenorientierten Alterssicherungspolitik: ${ }^{93}$ Ein stabiler, höchstens bis 22 Prozent steigender Sozialbeitragssatz sollte künftig Vorfahrt vor der Sicherung des Rentenniveaus haben. Die zweite Strukturreform führte eine ergänzende freiwillige kapitalgedeckte, staatlich subventionierte Rente als dritte Säule der Alterssicherung ein, die nach dem damaligen Bundesarbeitsminister Walter Riester benannte „Riester-Rente“. ${ }^{94}$ Sie sollte die Alterssicherung auf die Herausforderungen einstellen, die durch die Alterung der Bevölkerung, die Finanzierungsengpässe der Rentenkassen, die Konflikte zwischen der Sozialstaatsfinanzierung und wirtschaftspolitischen Anreizen sowie die mittelfristig voraussichtlich zunehmende Altersarmut gegeben sind.

Noch mehr Aufsehen erregten jene vier Arbeitsmarktreformen, die die rot-grüne Regierung im Rahmen des Reformprogramms „Agenda 2010” unter Gerhard Schröder im März 2003 auf den Weg brachte. Diese Arbeitsmarktreformen wur-

91 Bundesministerium für Arbeit und Sozialordnung (Hg.): Statistische Übersichten zur Geschichte der Sozialpolitik in Deutschland seit 1945 - Band West. Verfasser Hermann Berié, Bonn, 1999, 24; vgl. Schmidt, M.G.: Bundesrepublik Deutschland 1982-1989, a.a.O.

92 Sachverständigenrat zur Begutachtung der gesamtwirtschaftlichen Entwicklung: Jahresgutachten 1997/98, Stuttgart u.a. 1997, Ziffer 239.

93 Alber, J.: Recent Developments in the German Welfare State: Basic Continuity or a Paradigm Shift?, in: Gilbert N./van Voorhis, R.A. (Hg), Changing Patterns of Social Protection,, New Brunswick NJ, 2003, 9-73, hier $32 \mathrm{ff}$.

94 Trampusch, C.: Der erschöpfte Sozialstaat. Transformation eines Politikfeldes, Frankfurt a.M., 2009; Jochem, S.: Reformpolitik im Wohlfahrtsstaat. Deutschland im internationalen Vergleich, Berlin, 2009, $262 \mathrm{ff}$. 
den als „Hartz-Reformen“ bekannt - benannt nach dem damaligen VWPersonalchef Peter Hartz, einem Berater der Regierung Schröder. ${ }^{95}$

Die am weitesten reichende Arbeitsmarktreform war „Hartz IV“, am 23.12.2003 beschlossen und rund 12 Monate später, am 1.1.2005, in Kraft getreten. „Hartz IV" schuf eine Grundsicherung für Arbeitsuchende. Hierzu wurde die Sozialhilfe mit der Arbeitslosenhilfe zusammengelegt und die Arbeitslosenhilfe durch das als Fürsorgeleistung erbrachte „Arbeitslosengeld II“ bzw. das „Sozialgeld“ ersetzt, die beide - wie zuvor die Sozialhilfe - nach Bedürftigkeitsprüfung gewährt werden. Zudem wurde die maximale Bezugsdauer des Arbeitslosengeldes auf 12 bis 18 Monate abgesenkt. Diese Reform verstärkte den Anreiz zur Arbeitsaufnahme - und verhängte bei Zuwiderhandlung Sanktionen wie Absenkung der Sozialleistungen. Hinzu traten strengere Kriterien für zumutbare Arbeit.

„Hartz IV“ war eine Strukturreform, eine Reform dritter Ordnung ${ }^{96}$ : Sie veränderte die vorhandenen Stellschrauben der Arbeitsmarktpolitik, führte neue arbeitsmarktpolitische Instrumente ein und legte eine neue Regelungsphilosophie fest, indem sie den Grundgedanken des ,aktivierenden Sozialstaates“ - Fördern und Fordern - auf die Grundsicherung für Arbeitssuchende anwandte.

Die Arbeitsmarktreformen Hartz I bis IV flexibilisierten den ohnehin schon von „Liberalisierungspolitik“97 geprägten Arbeitsmarkt noch weiter. Entsprechend hart fiel die Kritik des linken Spektrums aus: „Schleifung von Arbeitnehmerschutzrechten“, „Sozialabbau“, „Rekommodifizierung“ statt „Dekommodifizie-

95 Trampusch, C., a.a.O.; Jochem, S.: Reformpolitik im Wohlfahrtsstaat, a.a.O., 238 ff. „Hartz I“ - beschlossen am 23.12.2002, in Kraft ab 1.1.2003 - erleichterte die Leiharbeit durch Gründung sogenannter Personal Service Agenturen (PSA), lockerte die Bindung des Arbeitslosengeldes und der Arbeitslosenhilfe von der Lohnentwicklung und regelte die Kriterien für zumutbare Beschäftigung von Arbeitslosen. „Hartz II“, ebenfalls am 23.12.2002 beschlossen und ab 1.1.2003 in Kraft, beseitigte den bürokratischen Überschwang der Sozialpolitik der ersten rot-grünen Wahlperiode durch eine beschäftigungsfreundlichere Reform der geringfügigen Beschäftigung, die Aufhebung der Regelungen zur „Scheinselbstständigkeit“ und die Einrichtung von „Ich-AGs“ als neuer Form der Existenzgründung. „Hartz III“ (beschlossen am 23.12.2003 und in Kraft seit 1.1.2004) stieß ins Zentrum der korporatistischen Verwaltung der Arbeitsmarktpolitik, die durch geschönte Vermittlungsbilanzen der Nürnberger Bundesanstalt für Arbeit in die Kritik geraten war, und leitete eine Reform der Arbeitsmarktverwaltung ein. Die „Bundesagentur für Arbeit“, so die neue Bezeichnung für die ehemalige Bundesanstalt für Arbeit seit 1.1.2004, sollte ihre Tätigkeit auf ihre Kernaufgabe, die effiziente und effektive Vermittlung von Arbeitslosen konzentrieren und nicht länger wie die Bundesanstalt für Arbeit als ein arbeits- und sozialpolitischer „Gemischtwarenladen“, als „Bundesanstalt für Alles“ wirken.

96 Hall, P.A.: Social Learning, and the State. The Case of Economic Policymaking in Britain, in: Comparative Politics, 25/2, 1993, 275-296.

97 Höpner et al. 2011, a.a.O. 
rung“ waren die Schlüsselparolen. Entsprechend lebhaft war andererseits die Zustimmung zu den Arbeitsmarktreformen auf bürgerlich-liberaler Seite, aber auch bei Fachleuten der Arbeitsmarkt- und Wirtschaftspolitik. ${ }^{98}$

Doch nicht nur mit spektakulären Reformen wie Hartz I bis IV und RiesterRente wartete Deutschlands Sozialpolitik bei der Anpassung an neue Herausforderungen auf. Hinzu traten inkrementelle Korrekturen insbesondere an den Leistungen der Alterssicherung, die sich zu einer beträchtlichen finanziellen Konsolidierung häuften.

Davon zeugen die öffentlichen Ausgaben für Alter und Hinterbliebene. ${ }^{99}$ Sie sind hoch, wie der deutschlandinterne Längsschnitt ebenso zeigt wie der internationale Vergleich. Allerdings hängen Deutschlands überdurchschnittlich hohe Alterssicherungsausgaben eng mit der weit fortgeschrittenen Alterung seiner Bevölkerung zusammen - und sind deshalb nicht nur ein Ergebnis großzügiger Sozialpolitik. Zudem gibt es berichtenswerte trends: Der Anteil der Alterssicherungsausgaben am Bruttoinlandsprodukt in Deutschland nimmt seit dem Höchststand von 2003 wieder ab - und dies in einer Zeit steigender Seniorenquoten. ${ }^{100}$

Der internationale Vergleich der Alterssicherungsausgaben unterstützt diesen Befund: Bis zur Jahrtausendwende lag Deutschland - gemessen am Zusammenhang von Alterssicherungsausgaben und Seniorenquote in allen OECDMitgliedstaaten - oberhalb der Trendlinie. Inzwischen aber ist Deutschland auf oder unter dieser Linie positioniert. Die Alterssicherungsausgaben in Deutschland waren demnach bis etwa 2000 höher als sie gemäß dem in den OECDLändern herrschenden Trend hätten sein müssen. Inzwischen aber sind sie niedriger - trotz eines überdurchschnittlich hohen Anteils von Rentnern und Pensionären. Das ist ein belastbarer Hinweis auf eine beachtliche Anpassung der Finanzen für die Alterssicherungssysteme. Bedarfsgewichtete Sozialleistungsquoten deuten in die gleiche Richtung. Gemessen am Bevölkerungsanteil der Senioren blieben die Alterssicherungsausgaben in Deutschland in den 1980er Jahren und

98 Vgl. Sachverständigenrat für die Begutachtung der gesamtwirtschaftlichen Entwicklung: Erfolge im Ausland - Herausforderungen im Inland. Jahresgutachten 2004/05, Wiesbaden 2004, $187 \mathrm{ff}$.

99 Vgl. ausführlicher Schmidt, M.G.: Die Demokratie wird älter - Politische Konsequenzen des demographischen Wandels, in: Kielmansegg, P. Graf/Häfner, H. (Hg.): Alter und Altern. Wirklichkeiten und Deutungen, Heidelberg, 2012, 163-186.

100 Berechnet auf der Basis von BMAS: Statistisches Taschenbuch 2010. Arbeits- und Sozialstatistik, Bonn, 2010, Tab. 7.2. 
in den beiden folgenden Jahrzehnten weiter zurück als in etlichen anderen Staaten. 101

Dass der Anteil der öffentlichen Ausgaben für Alter und Hinterbliebene in Deutschland inzwischen tendenziell sinkt - trotz hoher und weiter steigender Seniorenquote - ist bemerkenswert. Die Hauptursache ist eine Serie von teils kleineren, bisweilen mittelgroßen, seltener großen Umbau- und Rückbaureformen in der Alterssicherung, die sich in spürbaren Kürzungen im Bereich der gesetzlichen Rentenversicherung und anschließend in der Alterssicherung für die Beamten niederschlugen. Korrekturen der Alterssicherungssysteme erfolgten, wenngleich noch zögerlich, in der zweiten Hälfte der 1970er Jahre: Damals wurden die Leistungen pro Empfänger erstmals abgesenkt. ${ }^{102}$ Stärker wirkten die Korrekturen der Rentenformel von 1977, 1982 und 1983 sowie zwei zeitlich verzögerte Rentenanpassungen. Gemessen an der Standardrente eines Durchschnittsverdieners mit 40-jähriger Versicherungszeit beliefen sich die Kürzungen allein beim Vergleich von 1984 mit 1977 auf rund 15 Prozent. ${ }^{103}$

Weitere Reformen der Alterssicherung mit in Teilen erheblichem Kürzungspotential kamen in den darauf folgenden Jahren hinzu - zunächst noch unter der Koalition aus CDU, CSU und FDP, anschließend unter Rot-Grün und sodann auch im Rahmen der zweiten Großen Koalition. ${ }^{104}$ Schulze und Jochem zufolge verminderte etwa die Rentenreform 1992 den Beitragssatz zur Alterssicherung nach einem Ablauf von zehn Jahren um 2,5 Prozentpunkte und nach weiteren 20 Jahren um 3,5 Prozentpunkte. Die Alterssicherungsreform 1999 hatte die Beitragssätze in den gleichen Zeiträumen um weitere zwei Prozentpunkte bzw. 2,6 Prozentpunkte reduziert. Und das Altersvermögensgesetz von 2001 wird voraussichtlich in beiden Berichtszeiträumen den Beitragssatz um jeweils einen Prozentpunkt absenken. ${ }^{105}$ Überdies wird die Erhöhung des Renteneintrittsalters auf 67 Jahre, die von der zweiten Großen Koalition (2005-2009) beschlossen wurde, die Rentenfinanzen ebenfalls entlasten.

101 Siegel, N.A., a.a.O., $146 \mathrm{ff}$.

102 Alber, J.: Der Sozialstaat in der Bundesrepublik 1950-1983, Frankfurt a.M.-New York, 1989, 249 f.

103 Alber, J.: Germany, in: Flora, P. (Hg.): Growth to Limits. The Western European Welfare States Since World War II, Bd. 2, Berlin-New York, 1986, 1-154, hier 120; ders.: Recent Developments, a.a.O., 32 ff.

104 OECD, Employment Outlook, 2007, a.a.O., $59 \mathrm{ff}$.

105 Schulze, I./Jochem, S.: Germany: beyond policy gridlock, in: Immergut E.M./Anderson, K.M./Schulze, I. (Hg.): The Handbook of West European Pension Politics, Oxford, 2007, 660-710, Zitat 705, Anm. 26. 
Insoweit überrascht es nicht, wenn Experten der Politik in Deutschland bescheinigen, sie sei „,bei der Eindämmung der Alterssicherungskosten recht erfolgreich“"106 - trotz zahlreicher institutioneller Schranken sowie einflussreicher Vetospieler. Die Befunde der sozialpolitischen Umbau- und Rückbaumaßnahmen in Deutschland fügen sich, wie der internationale Vergleich zeigt, zu einem auffälligen Muster: Deutschland zählt inzwischen nicht nur in der Arbeitsmarktpolitik $\mathrm{zu}$ den Reformstaaten ${ }^{107}$, sondern auch in der Rentenpolitik - gemessen an den Anpassungen der Alterssicherungssysteme an den demographischen Wandel und andere Herausforderungen, wie schwächere wirtschaftliche Entwicklung und internationaler Standortwettbewerb. ${ }^{108}$ Dieses Leistungsprofil teilt Deutschland mit einigen anderen Ländern mit aufwendigen Alterssicherungssystemen (wie Schweden) und Staaten, in denen die Pensionssysteme frugaler dosiert sind.

Auch im internationalen Vergleich von Umbau- und Rückbaumaßnahmen erweist sich Deutschlands Sozialpolitik gemäß der in diesem Kapitel vorgelegten Befunde als besser als der Ruf, der ihr in einem einflussreichen Teil der vergleichenden Sozialpolitikforschung vorausgeht. Von Reformträgheit oder gar von anhaltendem Reformstau kann in Deutschlands Sozialpolitik nicht die Rede sein.

\section{Nebenwirkungen der Sozialpolitik}

Die Befunde der beiden letzten Kapitel stützen die These, dass Deutschlands Sozialpolitik Probleme bewältigen kann. ${ }^{109}$ Dass sie auch Probleme erzeugt und überdies schwere Nebenwirkungen hat, ist ebenfalls nicht zu übersehen. Drei dieser Nebenwirkungen werden hier erörtert, weil sie besonders ins Gewicht fallen und weil für sie ebenfalls neuere international vergleichbare Daten vorliegen: die Probleme der sozialstaatlichen Finanzierungskosten, die Zurückdrängung anderer ausgabenintensiver Politikfelder durch die Sozialpolitik sowie ihr Beitrag zur Staatsverschuldung.

Dass die Arbeitslosigkeit eine Achillesferse des deutschen Sozialstaats ist (vgl. unter V.), hat viele Ursachen. Eine davon ist die Art seiner Finanzierung. Weil

106 Schulze, I./Jochem, S., a.a.O., 697, Übersetzung d. Verf.

107 Jäkel, T./Hörisch, F.: Die Deregulierung von Arbeitsmärkten im OECD-Vergleich zwischen 1990 und 2005, in: Wagschal, U. (Hg.): Deutschland zwischen Reformstau und Veränderung. Ein Vergleich der Politik und Handlungsfelder, Baden-Baden, 2009, 83-104.

108 OECD, Pensions, 2007, a.a.O.; OECD, Pensions, 2009, a.a.O.

109 Das gilt im Übrigen auch hinsichtlich beachtlicher politischer und ökonomischer Stabilisierungsleistungen der Sozialpolitik. Vgl. Schmidt, M.G.: Sozialpolitik als Stabilisierungsfaktor der bundesrepublikanischen Entwicklung?, in: Zeitschrift für Politik, 56/4, 2009, 427-435. 
rund 60 Prozent der gesamten Sozialausgaben aus Sozialbeiträgen finanziert werden und der Anteil der Sozialbeiträge sowohl am Bruttolohn als auch am Bruttoinlandsprodukt dementsprechend überdurchschnittlich hoch ausfällt, ${ }^{110}$ wirkt der größte Teil der Sozialstaatsfinanzierung in Deutschland wie eine Sondersteuer auf den Faktor Arbeit. Das bremst die Beschäftigung, dämpft das Wirtschaftswachstum und schmälert damit die Einnahmenbasis des Sozialstaats unter sonst gleichen Bedingungen. Die Nebenwirkung hat allerdings auch eine produktive Seite: Die deutsche Variante der Sozialstaatsfinanzierung vergrößert den Anreiz für arbeitssparende Investitionen und fördert hierdurch die Modernisierung der Wirtschaft. Auf diese Weise wirkt der Sozialstaat als ökonomischer „Produktivfaktor“"111, der der langfristigen Entwicklung der Wirtschaft dienen kann.

Hinzu kommt eine zweite Nebenwirkung. In Staaten mit mittlerer gesamtwirtschaftlicher Abgabenquote - wie in Deutschland - drängt ein aufwendiges Sozialbudget andere ausgabenintensive Aufgabenfelder zurück, etwa das Bildungswesen. $^{112}$ Ein Grund dafür liegt in dem finanzierungspolitischen Vorteil der Sozialpolitik gegenüber weitgehend steuerfinanzierten Staatsaufgaben. Soweit sich die Sozialpolitik aus Sozialbeiträgen finanziert, nützt ihr die Aura des guten Zwecks, die den Sozialbeiträgen einschließlich ihrer Erhöhung vorausgeht. Zudem kann die Finanzierung durch Sozialbeiträge und gegebenenfalls ihre Erhöhung politisch einfacher durchgesetzt werden: Sie bedarf nicht der Zustimmung des Bundesrates.

Staatsaufgaben außerhalb der Sozialpolitik hingegen werden größtenteils aus Steuern finanziert, und oft erfordern sie Steuererhöhungen. Doch Steuern und Steuererhöhungen sind unpopulär. Obendrein sind sie politisch schwieriger $\mathrm{zu}$ bewerkstelligen, weil die gesetzliche Regulierung insbesondere der ertragreichsten Steuern der Zustimmung des Bundesrates unterliegt. Allein das erschwert die Finanzierung von Staatsaufgaben wie die Bildungspolitik. Denn die Bildungsetats werden überwiegend aus den - notorisch klammen - Länderhaushalten finanziert, die aufgrund der Abneigung gegen Steuererhöhungen und der scharfen Finanzierungskonkurrenz mit anderen Länderverpflichtungen, wie hohen

110 Obinger, H./Wagschal, U.: Social Expenditure and Revenues, a.a.O.

111 Die Formel entstammt der Selbstbeschreibung des deutschen Sozialstaats und meint vor allem seine politischen und gesellschaftlichen Stabilisierungsleistungen (BMAS 2012, a.a.O., 2).

112 Castles, F.G., a.a.O. 
Personal- und Pensionskosten, einen weit geringeren Finanzierungsspielraum als sozialabgabenfinanzierte Programme haben. ${ }^{113}$

In Deutschland sind die Finanzierungsschwierigkeiten für Politikfelder außerhalb der Sozialpolitik besonders groß: Hier werden die Staatsaufgaben außerhalb der Sozialpolitik größtenteils aus einem Steuervolumen finanziert, das im internationalen Vergleich nur unterdurchschnittliche Größe hat. ${ }^{114}$

Eine dritte große Nebenwirkung der Sozialpolitik ist diese: Ihr Auf- und Ausbau trugen zum Anstieg der Staatsverschuldung bei. Gewiss: Entgegen populistischer Diagnosen - wie der vom „Schuldenstaat“ der „Pleite-Republik““115 - gibt es keine Eins-zu-eins-Entsprechung zwischen Sozialausgaben und Staatsverschuldung. Doch der Anstieg der Staatsverschuldung in Deutschland von knapp 20 Prozent des Bruttoinlandsproduktes in den ausgehenden 1960er Jahren auf rund 80 Prozent im Jahre $2012^{116}$ kann ohne die Sozialfinanzen nicht erklärt werden.

\section{Schlussfolgerung}

Der vorliegende Beitrag zeigt, dass der deutsche Sozialstaat viel mehr ist als ein „Sozialversicherungsstaat“. Auch wird er weder als „,konservativer Wohlfahrtsstaat" angemessen beschrieben noch als ein Staat, der insgesamt in marktliberale Richtung treibt. Deutschlands Sozialstaat verkörpert einen - gegenüber den 1980er und 1990er Jahren - komplexeren Mischtyp. ${ }^{117}$ In Esping-Andersens Terminologie ist er eine Mixtur aus „konservativen“ und „sozialdemokratischen“ Komponenten. $\mathrm{Zu}$ ihm zählen außerdem liberale Regulierungsformen wie bedürftigkeitsgeprüfte Mindestsicherungssysteme, der Aufbau einer kapitalgedeckten Säule der Alterssicherung und ein Rückbau des Beschäftigungsschutzes für

113 Schmidt, M.G.: Die öffentlichen und privaten Bildungsausgaben in Deutschland im internationalen Vergleich, in: Zeitschrift für Europa- und Staatswissenschaften 2/1, 2004, 7-31; Wolf, F.: Bildungsfinanzierung in Deutschland, Wiesbaden, 2008.

114 Das ist die Kehrseite des starken Sozialabgabenstaates, vgl. OECD: Revenue Statistics 1965-2010, Paris, 2011, 83. Deutschlands gesamtwirtschaftliche Steuerquote (ohne Sozialbeiträge) betrug 2009 22,9 Prozent des BIP, während der OECD-Durchschnitt 24,6 Prozent erreichte, ebd.: 83; Wolf, a.a.O.

115 Hank, R.: Die Pleite-Republik - Wie der Schuldenstaat uns entmündigt und wie wir uns befreien können, München, 2012.

116 Sachverständigenrat zur Begutachtung der gesamtwirtschaftlichen Entwicklung: Die Zukunft nicht aufs Spiel setzen. Jahresgutachten 2009/10, Wiesbaden, 2009, 69, 373; Sachverständigenrat zur Begutachtung der gesamtwirtschaftlichen Entwicklung: Verantwortung für Europa wahrnehmen. Jahresgutachten 2011/11, Wiesbaden, 2011, 71.

$117 \mathrm{Zu}$ dessen Struktur und Werdegang Schmidt, M.G.: Der deutsche Sozialstaat. Geschichte und Gegenwart, München, 2012 (i.E.). 
befristete Jobs. Hinzu kommen Prinzipien, die der katholischen Soziallehre entstammen, wie das Subsidiaritätsprinzip.

Entgegen einer in der vergleichenden Sozialstaatsforschung weit verbreiteten Meinung, übertrifft das Leistungsprofil des deutschen Sozialstaats in vielerlei Hinsicht die Erwartungen und Diagnosen seiner Kritiker. Davon zeugen nicht nur hohe Bruttosozialausgaben und sehr hohe Nettosozialausgaben, sondern auch weit ausgebaute Mindestsicherungssysteme für knapp zehn Prozent der Bevölkerung, die Kombination von Sozialsystemen und Regulierung der Arbeitswelt, Fortschritte bei den Erwerbs- und Beschäftigungsquoten, eine erhebliche Eindämmung von Einkommensungleichheit und Armutsrisiken sowie eine Reihe weit reichender Sozialstaatsreformen erster, zweiter und dritter Ordnung.

Wie jede starke Medizin hat Deutschlands Sozialstaat beträchtliche Nebenwirkungen. Neben der Arbeitslosigkeit, immer noch seine Achillesferse, sind drei Nebenwirkungen von besonders großem Gewicht: Erstens lastet die Finanzierung aus Sozialbeiträgen als beschäftigungsdämpfende Zusatzsteuer auf dem Faktor Arbeit. Zweitens drängt die Sozialpolitik aufgrund von finanzierungspolitischen Vorteilen andere ausgabenintensive Aufgaben jenseits der Sozialpolitik zurück. Und drittens zählen Auf- und Ausbau der Sozialpolitik zu den Ursachen des Anstiegs der Staatsverschuldung auf rund 80 Prozent des Bruttoinlandsprodukts.

Schlussendlich ist eine eigentümliche Lücke in Deutschlands Sozialstaat zu bedenken: Es ist die Lücke zwischen einem - gemessen an den Sozialausgaben sehr hohen Sozialstaatsinput einerseits und mittelgradiger Generosität der Sozialleistungen pro Empfänger in den Kernsystemen der sozialen Sicherung andererseits, insbesondere in der Renten-, der Kranken- und der Arbeitslosenversicherung. Warum diese Lücke besteht, ist noch nicht zweifelsfrei geklärt. Viel spricht für die These, dass sie aus den besonderen Bedarfs- und Kostenstrukturen im Lande resultiert, zu denen die weit fortgeschrittene Alterung der Bevölkerung, die relativ hohe Arbeitslosigkeit, die Folgekosten der deutschen Einheit, kostentreibende Wirkungen der Mindestsicherungssysteme und eine sehr große Sozialstaatsklientel gehören. Es ist demnach die Breite und Vielfalt der Aufgaben des Sozialstaats und nicht etwa Mittelmäßigkeit, die die Lücke zwischen sehr hohen Sozialausgaben und mittelgradiger Generosität in den Kernsystemen der sozialen Sicherung in Deutschland erklärt. Die Ursachen dieser Konstellation sind vielfäl- 
tig. ${ }^{118}$ Aus ihnen ragt eine - auch im internationalen Vergleich auffällige - besonders sozialstaatsfreundliche Parteienlandschaft heraus, in der zwei große Sozialstaatsparteien - CDU/CSU und SPD - sowie eine kleinere radikalsozialistische Verfechterin des Wohlfahrtsstaates - die Partei Die Linke - mit ehrgeiziger Sozialpolitik um die Gunst der Wähler konkurrieren.

118 Vgl. hierzu ausführlich Schmidt, M.G., Sozialpolitik, a.a.O.; Schmidt, M.G./Ostheim, T./Siegel, N.A./Zohnlhöfer, R., a.a.O. 


\section{Anhang}

Tabelle 1: Auszug aus der Datengrundlage

\begin{tabular}{|c|c|c|c|c|c|c|c|c|c|c|c|c|c|c|c|}
\hline $\begin{array}{c}1 \\
\text { Land }\end{array}$ & $\begin{array}{c}2 \\
\text { Bsq } \\
\end{array}$ & \begin{tabular}{|c|}
3 \\
Nsq1
\end{tabular} & \begin{tabular}{|c|}
4 \\
$\mathrm{Nsq} 2$ \\
\end{tabular} & $\begin{array}{c}5 \\
\text { Dek } \\
\end{array}$ & \begin{tabular}{|c|}
6 \\
Gen
\end{tabular} & $\begin{array}{c}7 \\
\text { Eu }\end{array}$ & $\begin{array}{c}8 \\
\text { Eb } \\
\end{array}$ & \begin{tabular}{|c|}
9 \\
ME \\
\end{tabular} & \begin{tabular}{|l|}
10 \\
FE \\
\end{tabular} & $\begin{array}{c}11 \\
\text { Alq } \\
\end{array}$ & $\begin{array}{c}12 \\
\text { Arm }\end{array}$ & \begin{tabular}{|c|}
13 \\
Arm \\
\end{tabular} & \begin{tabular}{c|}
14 \\
Gini
\end{tabular} & \begin{tabular}{|c|}
15 \\
Baq
\end{tabular} & $\begin{array}{c}16 \\
\text { Staat } \\
\end{array}$ \\
\hline Australien & 16,0 & 18,3 & 21,0 & 13,0 & 19,4 & 1,5 & 0,9 & 82,9 & 70,0 & 5,2 & & $\mid-12,6$ & $-0,13$ & 4,3 & 51,3 \\
\hline Belgien & 26,3 & 26,2 & 30,5 & 32,4 & 31,0 & 1,7 & 2,6 & 73,4 & 61,8 & 8,3 & 44,4 & $-22,1$ & $-0,21$ & 6,0 & 54,3 \\
\hline Dänemark & 26,0 & 23,9 & 25,3 & 38,1 & 35,6 & 1,6 & 1,4 & 82,7 & 76,1 & 7,4 & 57,1 & $-16,0$ & $-0,16$ & 7,8 & 51,1 \\
\hline Deutschland & 25,2 & 27,2 & 28,4 & 27,7 & 28,6 & 3,0 & 1,3 & 82,4 & 70,8 & 7,1 & $\mathbf{5 0 , 0}$ & $-23,6$ & $-0,21$ & 4,5 & 57,7 \\
\hline Estland & 13,0 & & & & & 2,5 & 1,8 & 73,7 & 70,9 & 16,9 & 36,0 & $-14,1$ & $-0,14$ & 4,8 & 37,4 \\
\hline Finnland & 24,9 & 22,6 & 23,4 & 29,2 & 30,8 & 2,2 & 1,8 & 74,6 & 72,5 & 8,4 & 55,2 & $-22,5$ & $-0,21$ & 5,9 & 52,6 \\
\hline Frankreich & 28,4 & 29,9 & 32,7 & 27,5 & 29,3 & 2,5 & 3,6 & 70,6 & 66,3 & 9,8 & 48,0 & $-25,4$ & $-0,19$ & 5,6 & 54,3 \\
\hline Griechenland & 21,3 & & & & & 2,3 & 3,1 & 68,2 & 57,6 & 12,6 & 8,7 & $-20,3$ & $-0,13$ & & 48,0 \\
\hline Großbritannien & 20,5 & 22,7 & 26,9 & 23,4 & 20,0 & 1,1 & 0,4 & 76,3 & 70,2 & 7,8 & 36,7 & $-20,2$ & $\mid-0,11$ & 5,4 & 44,2 \\
\hline Irland & 16,3 & 16,8 & 18,0 & 23,3 & 22,4 & 1,6 & 0,6 & 70,2 & 62,6 & 13,7 & 45,5 & & & 4,9 & 45,0 \\
\hline Island & 14,6 & 16,8 & 19,7 & & & 1,7 & 0,6 & 85,5 & 82,7 & 7,5 & & & $-0,08$ & 7,4 & 34,4 \\
\hline Italien & 24,9 & 25,8 & 26,4 & 24,1 & 21,7 & 1,8 & 2,0 & 62,2 & 51,1 & 8,4 & 16,7 & $-21,9$ & $-0,20$ & 4,3 & 52,0 \\
\hline Japan & 18,7 & 20,3 & 23,4 & 22,3 & 17,4 & 1,9 & 1,0 & 74,0 & 63,2 & 5,1 & & $\mid-13,0$ & $-0,13$ & 3,4 & 50,8 \\
\hline Kanada & 16,9 & 19,4 & 24,0 & 22,0 & 23,9 & 1,3 & 0,3 & 81,5 & 74,2 & 8,0 & & $-12,7$ & $-0,12$ & 4,9 & 42,9 \\
\hline Korea & 7,6 & 9,5 & 11,8 & & & 2,4 & 1,4 & 65,8 & 54,5 & 3,7 & & $-2,5$ & $-0,07$ & & 26,5 \\
\hline Luxemburg & 20,6 & 19,1 & 19,6 & & & 2,8 & 3,8 & 68,2 & 60,3 & 4,5 & 41,7 & $-18,4$ & $-0,19$ & & 56,9 \\
\hline Mexiko & 7,2 & 8,9 & 9,0 & & & 2,3 & 4,0 & 63,9 & 46,6 & 5,4 & & $-2,6$ & $-0,02$ & 4,8 & 32,1 \\
\hline Neuseeland & 18,4 & 18,4 & 18,8 & 17,1 & 25,5 & 1,6 & 1,3 & 77,5 & 71,8 & 6,5 & & $-11,4$ & $-0,13$ & 5,8 & 57,3 \\
\hline Niederlande & 20,1 & 20,4 & 25,3 & 32,4 & 34,9 & 2,7 & 1,2 & 78,2 & 72,6 & 4,5 & 52,4 & $-16,9$ & $-0,14$ & 5,3 & 44,2 \\
\hline Norwegen & 20,8 & 20,0 & 20,5 & 38,3 & 38,2 & 2,3 & 3,1 & 78,2 & 75,6 & 3,5 & & $-16,0$ & $-0,16$ & 6,7 & 50,7 \\
\hline Österreich & 26,4 & 24,8 & 25,8 & 31,1 & 27,3 & 2,9 & 1,5 & 80,9 & 69,3 & 4,4 & 48,0 & $-20,9$ & $-0,21$ & 5,4 & 54,1 \\
\hline Polen & 19,8 & 18,6 & 18,6 & & & 2,1 & 1,8 & 65,6 & 59,0 & 9,7 & 34,5 & $-16,5$ & $-0,16$ & 4,9 & 46,9 \\
\hline Portugal & 22,5 & 23,6 & 25,0 & & & 4,2 & 2,1 & 74,0 & 69,9 & 11,0 & 28,0 & $-16,4$ & $-0,18$ & 5,3 & 49,1 \\
\hline Schweden & 27,3 & 25,9 & 27,8 & 39,1 & 40,8 & 2,9 & 0,9 & 79,5 & 76,7 & 8,4 & 58,6 & $-18,1$ & $-0,17$ & 6,7 & 52,0 \\
\hline Schweiz & 18,5 & & & 29,8 & 26,1 & 1,2 & 1,1 & 82,2 & 76,1 & 4,2 & & $-10,6$ & $-0,11$ & 5,2 & 43,5 \\
\hline Slowakei & 15,7 & 16,0 & 16,7 & & & 2,5 & 0,4 & 68,7 & 61,3 & 14,4 & 40,0 & $-17,5$ & $-0,16$ & 3,6 & 84,4 \\
\hline Slowenien & 20,3 & & 0 & & & 3,2 & 1,9 & 71,5 & 67,4 & 7,3 & 50,0 & $-17,3$ & $\mid-0,19$ & 5,2 & 47,9 \\
\hline Spanien & 21,6 & 21,6 & 21,8 & & & 2,5 & 3,5 & 74,4 & 66,8 & 20,1 & 16,7 & $-13,2$ & $-0,14$ & 4,3 & 55,1 \\
\hline Tschechien & 18,8 & 19,2 & 19,3 & & r & 3,1 & 0,9 & 78,6 & 61,5 & 7,3 & 54,6 & $-20,1$ & $-0,19$ & 4,2 & 44,2 \\
\hline Türkei & 10,5 & 11,3 & 11,3 & & & 2,6 & 4,9 & 52,7 & 30,2 & 10,7 & & $\mid-5,0$ & $-0,06$ & & \\
\hline Ungarn & 22,9 & & & & & 1,9 & 1,4 & 62,4 & 56,7 & 11,2 & 46,7 & $-20,2$ & $-0,19$ & 5,2 & 46,0 \\
\hline USA & 16,2 & 18,9 & 27,5 & 14,2 & 18,5 & 0,2 & 0,3 & 73,9 & 68,4 & 9,6 & & $\mid-9,7$ & $-0,11$ & 5,3 & 43,5 \\
\hline Durchschnitt & 19,6 & 19,5 & 21,4 & 26,9 & 27,3 & 2,2 & 1,8 & 73,6 & 65,5 & 8,5 & $\overline{41,4}$ & $-15,9$ & $-0,15$ & 5,3 & 48,7 \\
\hline
\end{tabular}

Fettsetzung: Überdurchschnittliche Performanz Deutschlands.

Spaltenbezeichnungen:

1. Ländername.

2. Bruttosozialleistungsquote 2007, Adema, W. et al., a.a.O., Tabelle A.I.1.3.

3. Nettosozialleistungsquote (ohne freiwillige Sozialleistungen) 2007, Adema, $W$. et al., a.a.O., Tabelle A.I.1.3.

4. Nettosozialleistungsquote (einschließlich freiwilliger Sozialleistungen) 2007, Adema, $W$. et al., a.a.O., Tabelle A.I.1.3.

5. Dekommodifizierungsindex nach Esping-Andersen, G: Three Worlds, a.a.O., $50,52$.

6. Sozialpolitische Generosität („overall generosity score“), Scruggs, L.: Benefit Generosity Indices, Excel-Datei 2.4.2006, Abruf 15.2.2012. 
7. Beschäftigungsschutz für unbefristete Beschäftigungsverhältnisse 2008 (Skala von 0 - Minimum - bis 6); Venn, D., a.a.O., 2009, 8 f.; www. oecd.org/employment/key statistics - Tabelle Employment Protection, Abruf am 9.1.2012.

8. Beschäftigungsschutz für befristete Beschäftigungsverhältnisse (Skala von 0 bis 6) 2008, Venn, D., a.a.O., 2009, 8f.; www.oecd.org/employment/key statistics - Tabelle Employment Protection, Stand 9.1.2012.

9. Männererwerbsquote 2010 (in Prozent der 15-64-Jährigen), $O E C D$ : OECD Employment Outlook 2011, a.a.O., 240.

10. Frauenerwerbsquote 2010 (in Prozent der 15-64-Jährigen), OECD: OECD Employment Outlook 2011, a.a.O., 241.

11. Arbeitslosenquote (in Prozent zivile Erwerbsbevölkerung) 2010, OECD: OECD Employment Outlook 2011, a.a.O., 238.

12. Differenz zwischen Armutsrisiko vor und nach Sozialtransfers (2005). Quelle: 3. Reichtumsund Armutsbericht der Bundesregierung (a.a.O.), XII und 328.

13. Differenz der Armutsrate (50\% des laufenden Medianeinkommens) vor und nach Steuer und Transferzahlungen ca. 2009 (nach Zahl der Personen angepasstes Haushaltseinkommen). Quelle: $O E C D$, Income Distribution Database (http://dx.doi.org/10.1787/888932563856, Abruf 11.3.2012).

14. Verminderung der Einkommensungleichheit durch Steuern und Sozialtransfer (Differenz der Gini-Indizes der Einkommensverteilung vor und nach Steuern und Transferzahlungen), 2009 oder nächstliegendes Jahr. Quelle: OECD, Income Distribution Database (http://dx.doi.org/10.1787/888932563856), Abruf 11.3.2012.

15. Öffentliche Bildungsausgaben einschließlich Subventionen an private Haushalte und andere private Einrichtungen in Prozent BIP 2007, OECD: Education at a Glance 2010, a.a.O., 243.

16. Bruttosozialausgaben in Prozent aller Staatsausgaben 2007. Quelle: Spalte 2, OECD: OECD at a Glance 2010, Paris, 2010, 454. 